\title{
GEOPHYSICS
}

\section{Multiscale Reflection Phase Inversion with Migration Deconvolution}

\begin{tabular}{|r|l|}
\hline Journal: & Geophysics \\
\hline Manuscript ID & GEO-2018-0751.R3 \\
\hline Manuscript Type: & Technical Paper \\
\hline Keywords: & reflection, phase, inversion, migration \\
\hline Area of Expertise: & Seismic Inversion, Seismic Migration \\
\hline &
\end{tabular}

\section{SCHOLARONE" Manuscripts}




\title{
Multiscale Reflection Phase Inversion with Migration Deconvolution
}

\author{
Yuqing Chen*, Zongcai Feng*, Lei Fu ${ }^{\dagger}$, Abdullah AITheyab ${ }^{\ddagger}$, Shihang Feng* and Gerard Schuster* \\ * Department of Earth Science and Engineering, \\ King Abdullah University of Science and Technology, \\ Thuwal, Saudi Arabia, 23955-6900. \\ Email: yuqing.chen@kaust.edu.sa \\ ${ }^{\dagger}$ Department of Earth Science and Space Sciences, \\ Southern University of Science and Technology, \\ Shen Zhen, China, 518055 \\ ¥ Saudi Aramco, Dhahran, Saudi Arabia, 31311,
}

(September 5, 2019)

Multiscale Reflection Phase Inversion with Migration Deconvolution

Running head: $\mathbf{M R P I}+\boldsymbol{M D}$

\begin{abstract}
Reflection full waveform inversion (RFWI) can recover the low-wavenumber components of the velocity model along with the reflection wavepaths. However, this requires an expensive least-square reverse time migration (LSRTM) to construct the perturbation image that can still suffer from cycle-skipping problems. As an inexpensive alternative to LSRTM, we propose the use of migration deconvolution (MD) with RFWI. To mitigate cycle-skipping problems, we introduce a multiscale reflection phase inversion (MRPI) strategy which boosts the low-frequency data and only needs to explain the phase information in the recorded data, not its magnitude spectrum. To mitigate cycle-skipping problems, we use the rolling-offset strategy which
\end{abstract}


gradually extends the offset range of data with an increasing number of iterations. Numerical results show that the MRPI + MD method can efficiently recover the low-wavenumber components of the velocity model and is less prone to getting stuck in local minima compared to conventional RFWI. 


\section{INTRODUCTION}

Full waveform inversion (FWI) has been shown to accurately invert seismic data for high-resolution estimates of the Earth's velocity distribution (Lailly and Bednar, 1983; Tarantola, 1984; Virieux and Operto, 2009). However, the success of FWI heavily relies on the availability of either low-frequency or long-offset diving waves and refractions in the seismic data (Xu et al., 2012; Wang et al., 2013; Fu and Symes, 2017) With a narrow-aperture acquisition geometry, FWI can only reconstruct the high-wavenumber components of the deep-velocity model illuminated by the deep reflections. Deep reflections recorded over a narrow aperture are unable to recover the long-wavelength components of the velocity model (Wang et al., 2013). To remedy this problem, Xu et al. (2012) developed a reflection full waveform inversion (RFWI) method that aims to retrieve the low-wavenumber components of the velocity model. RFWI splits the velocity model into a background (low-wavenumber component) and a perturbation model (high-wavenumber component). These two components are alternately updated at each iteration. For example, RFWI first computes the perturbed velocity model using the current background model. The calculated migration image is then used for Born modeling of the reflection data. The residuals between the Born-modeled traces and the observed traces are computed, which are then backpropagated to generate the source- and receiver-side reflection wavepaths for updating the background velocity model (Xu et al., 2012; Wang et al., 2013; Brossier et al., 2015).

Conventional RFWI requires a least-squares migration image with balanced amplitudes and high-resolution interfaces. However, LSRTM is computationally expensive because it requires a large number of iterations to get the desired uplift of image quality. To alleviate this expense, we use migration deconvolution (MD) (Hu and Schuster, 1998; Aoki and Schuster, 2009) as an inexpensive alternative of LSRTM, which can produce a migration image with image quality comparable to LSRTM without iterations. Migration deconvolution can efficiently approximate the Hessian inverse which is essential for an efficient improvement in 
image quality. Hu and Schuster (1998), Hu et al. (2001) and Yu et al. (2006) designed a MD operator in the space-wavenumber domain to suppress the migration artifacts. As an alternative, Guitton (2004) and Aoki and Schuster (2009) computed matching filters to construct a localized MD operator in the space domain, which is denoted as a deblurring filter in Aoki and Schuster (2009). In this paper, we follow the approach from Aoki and Schuster (2009) where the MD filters are computed directly in the space domain, while Guitton (2004) requires a demigration and migration of the original migration image. Chen et al. (2017) and Feng et al. (2018) extended the deblurring filter to the viscoacoustic and elastic cases, respectively. Feng and Schuster (2019) applied the deblurring filter to construct the zero-offset sections from time migration images.

Conventional RFWI uses the waveform-difference misfit function which is more likely to fall into local minima for poor starting models. This is because the misfit function is highly nonlinear and is very sensitive to the amplitude mismatch between the synthetic and the observed data. To alleviate these problems, Chi et al. (2015) developed a correlation-based misfit function which measures the kinematic differences between the observed and synthetic reflection events. Similarly, Feng and Schuster (2019) used the traveltime-difference misfit function to invert for both velocity and anisotropic parameters. Guo and Alkhalifah (2017) incorporated the elastic properties of RFWI to invert for both the P- and S-wave velocities. Wang et al. (2017) combined the elastic properties and the traveltime-based misfit function together to update both P- and S-wave velocities. Bozdağ et al. (2011), Wu et al. (2014) and Chen et al. (2018) introduced a reflection multiscale envelope inversion method so that the signal's envelope carries ultra-low frequency information missing in the original signal. Furthermore, a set of methods have been developed to exclude the cycle-skipped events in different domains (Bunks et al., 1995; Asnaashari et al., 2012; Bi and Lin, 2014; AlTheyab and Schuster, 2015).

In this paper, we employ a multiscale reflection phase inversion (MRPI) strategy to mitigate cycleskipping problems in conventional RFWI. We temporally integrate the seismic traces several times to both 
attenuate its high-frequency components and boost its low-frequency information. These low-boosted seismograms tend to mitigate cycle-skipping problems so that the solution is less prone to getting stuck in a local minimum. We then replace the magnitude spectrum of the synthetic data with that of the observed data. This modification relieves the inversion procedure of having to explain the magnitude spectrum in the observed data. Therefore, the misfit function focuses more on the phase mismatch than on the amplitude mismatch (Sun and Schuster, 1993; Fu et al., 2017). This phase mismatch is related to the kinematic errors associated with the low-wavenumber components of the velocity model.

The problem with multiscale phase inversion described above is that the far-offset reflection traces can still suffer from cycle-skipping problems. To mitigate this problem, we also apply the rolling-offset strategy of AlTheyab and Schuster (2015). In the rolling-offset strategy, we only use the near-offset traces at the early iterations that are not cycle-skipped, and later iterations gradually include larger offset traces into the migration and inversion. We also gradually reduce the order of the temporal integration to introduce higher-frequency information into the inverted model. As will be seen with our numerical examples, these strategies increase the effectiveness and robustness of reflection inversion.

This paper is organized into the following sections. After the introduction, we show how to incorporate migration deconvolution with multiscale reflection phase inversion. We then present the numerical results for both synthetic and $\mathrm{OBC}$ data from the North Sea. The field data presents a challenge for estimating the low-wavenumber parts of the velocity model. The last section provides a summary and conclusions. Appendix provides a detailed derivation of the imaging condition of the multiscale reflection phase inversion using the adjoint-state method. 


\section{THEORY}

Reflection full waveform inversion (RFWI) splits the subsurface velocity model $c$ into a background model (low-wavenumber component) $c_{0}$ and a perturbation model (high-wavenumber component) $\delta c$ (Xu et al., 2012)

$$
c=c_{0}+\delta c
$$

At each iteration, a migration image for $\delta c$ is first calculated under the current background model $c_{0}$. The estimated migration image $\delta c$ is then used for Born modeling of reflection data. RFWI computes the data residual by subtracting the Born data and recorded traces. The resulting residual traces are then backpropagated to generate the source- and receiver-side reflection wavepaths. These wavepaths are used to update the low-wavenumber components of the velocity model $c_{0}$, while $c_{0}$ and $\delta c$ are alternatively updated as the iterations proceed.

\section{Migration Deconvolution}

A migration image with balanced amplitudes and sharp interfaces is essential for generating strong reflection wavepaths. We use Born modeling to generate 100 shot gathers based on the true reflectivity and velocity model shown in Figure 1a-b, respectively. Figure 1c shows a reverse time migration (RTM) image with an illumination problem, in which the reflector amplitudes in the central region are much higher than in the side region. Figure 1e shows the least-squares reverse time migration (LSRTM) image at the 6th iteration, which has a more balanced amplitudes and a sharper interface compared to the RTM image. The RFWI gradients computed from the RTM and LSRTM images are shown in Figures $1 \mathrm{~d}$ and 1f, respectively. It shows that the RFWI gradient generated from the LSRTM image is more suited to update the low-wavenumber parts of the velocity model, because it well recovers the Gaussian anomaly that also appears in the true model shown 
in Figure 1b. Despite its accuracy in reconstructing the correct velocity model, LSRTM is computationally expensive because it requires many iterations to get the desired uplift in image quality. However, the problem with LSRTM is that it is more than an order-of-magnitude more expensive than standard RTM. To reduce this cost, migration deconvolution can provide image quality similar to LSRTM (see Figure 1g) but at the cost of about 1 RTM. As an example, Figure $1 \mathrm{~h}$ shows the accurate recovery of the central velocity anomaly using MD with RFWI. Therefore, we use the deblurring filter as our MD operator to compute the migration image for RFWI.

\section{Amplitude Replacement}

Currently, acoustic waveform inversion is the most widely used inversion method in the industry due to its low-computation costs. However, the isotropic acoustic assumption neglects elastic, attenuation, and anisotropic effects, which can severely compromise the inversion results. In some cases, a matching filter can be used to reduce the non-acoustic effects of the seismic data (Agudo et al., 2018), but the matching filters need to be iteratively updated and are prone to errors.

Sun and Schuster (1993) proposed amplitude replacement as a one-step method for compensating for some of the non-acoustic effects in the data. To eliminate the need to explain the magnitude spectrum, the magnitude spectra of the synthetic traces are replaced by those from the corresponding recorded traces. For any point source at $\mathbf{x}_{s}$ and a receiver at $\mathbf{x}_{r}$, both the observed traces $d_{o b s}\left(\mathbf{x}_{r}, t \mid \mathbf{x}_{s}\right)$ and the synthetic traces $d\left(\mathbf{x}_{r}, t \mid \mathbf{x}_{s}\right)$ are Fourier transformed into the frequency domain to obtain

$$
\begin{gathered}
D_{o b s}\left(\mathbf{x}_{r} \mid \mathbf{x}_{s}\right)=\mathscr{F}\left(d_{o b s}\left(\mathbf{x}_{r}, t \mid \mathbf{x}_{s}\right)\right)=A_{o b s}\left(\mathbf{x}_{r}, \omega ; \mathbf{x}_{s}\right) \exp \left[i \theta_{o b s}\left(\mathbf{x}_{r}, \omega ; \mathbf{x}_{s}\right)\right] \\
D\left(\mathbf{x}_{r} \mid \mathbf{x}_{s}\right)=\mathscr{F}\left(d\left(\mathbf{x}_{r}, t \mid \mathbf{x}_{s}\right)\right)=A\left(\mathbf{x}_{r}, \omega ; \mathbf{x}_{s}\right) \exp \left[i \theta\left(\mathbf{x}_{r}, \omega ; \mathbf{x}_{s}\right)\right],
\end{gathered}
$$


where $A$ and $\theta$ represent the magnitude and phase spectra, respectively, and $\mathscr{F}$ denotes the Fourier transform operator in the time domain. We replace the magnitude spectrum of the calculated data $A$ with that of the observed data $A_{o b s}$ and then apply the inverse Fourier transform to get the modified data:

$$
\tilde{d}\left(\mathbf{x}_{r}, t \mid \mathbf{x}_{s}\right)=\mathscr{F}^{-1}\left\{\tilde{D}\left(\mathbf{x}_{r} \mid \mathbf{x}_{s}\right)\right\}=\mathscr{F}^{-1}\left\{A_{o b s}\left(\mathbf{x}_{r}, \omega ; \mathbf{x}_{s}\right) \exp \left[i \theta\left(\mathbf{x}_{r}, \omega ; \mathbf{x}_{s}\right)\right]\right\}
$$

Therefore, there is only the phase mismatch between the observed and modified synthetic data. This phase mismatch is mostly related to the kinematic errors controlled by the low-wavenumber components of the velocity model.

As an example, Figures 2a-c show the acoustic, viscoacoustic, and elastic data, respectively. It clearly shows that the acoustic data do not contain the amplitude and phase distortions observed in the viscoacoustic data or the converted waves in the elastic data. Figures $2 \mathrm{e}$ and $2 \mathrm{f}$ show the acoustic traces where the magnitude spectra are replaced by the viscoacoustic and elastic data, respectively. The data residuals for the acoustic and viscoacoustic data with and without the amplitude replacement are compared in Figures 3a-b, respectively. They clearly show that the subtraction errors due to the viscoacoustic effects in the seismic data are largely reduced in Figure 3b. Similarly, the errors in the data residuals for the acoustic and elastic data are also greatly reduced after amplitude replacement when compared with Figure 3d with Figure 3c.

One limitation of this method is that it can't mitigate the phase mismatch caused by other physics such as attenuation and anisotropy, which may lead to overestimation of the velocity model. To mitigate this problem, we should introduce more physics to achieve a multi-parameter reflection phase inversion. In that case, the multi-parameter Hessian needs to be estimated in order to reduce the crosstalk between different parameter updates (Pan et al., 2018a,b; Feng et al., 2018). 


\section{Time Integration}

Time integration of a signal can be viewed as applying a low-boost filter to this signal (Tavares, 1966). Integrating a signal in the time domain is equivalent to dividing the spectrum by $i 2 \pi f$ in the frequency domain, and so boosts the low-frequency components and suppresses the high-frequency components of a signal. Figures $4 \mathrm{a}-\mathrm{c}$ show the traces with zero, one and two integrations, respectively, and Figure 5 shows their corresponding magnitude spectra. As the number of integrations increases, the high-frequency components are gradually attenuated and the low-frequency information dominates. Therefore, the recorded traces are integrated several times to boost their low-frequency components so that the integrated residual traces are less prone to be cycle-skipped (Fu et al., 2017). This is very similar to the multiscale inversion approach using bandpass filters (Bunks et al., 1995), except integration is in the time-domain method. However, the problem with a bandpass filter is that the bandpassed signal can be contaminated by artifacts generated by the narrow bandwidth and steep boundary of the bandpass filter. In comparison, the time integration operator does not have such effects. One disadvantage of the time integration, however, is that it might also boost the direct current (DC) term of the signal. But this problem can be eliminated by demeaning the data. In practice, we only integrate the source wavelet instead of integrating the synthetic data (see Appendix A).

\section{Rolling Offset}

For a poor starting model, simultaneous inversion of the full-offset range of data can lead to two problems: (1) far-offset data are more likely to be cycle-skipped compared with near-offset data because of the longer wavepaths; and (2) at the near-offset region, the amplitude and phase of the Born data do not agree with the observed data. The reason is that the stacked traces in the migration image becomes increasingly out-ofphase with wider offsets. This is because an incorrect migration velocity will adjust the reflector depth so that the near-offset synthetic traces match those from the field data, but this will not happen with increasing 
source-receiver offsets. Therefore, the inversion strategy is to first adjust the velocity model to first explain the near-offset data and then gradually adjust the velocity model to explain the traces with increasing offset. In this way, we can mitigate getting stuck in local minima and, hopefully, converge to the global minimum.

To numerically demonstrate the importance and advantages of the rolling-offset strategy, we test it on the two-layer model shown in Figure 6, where the upper- and bottom-layer velocities are $1800 \mathrm{~m} / \mathrm{s}$ and 2300 $\mathrm{m} / \mathrm{s}$, respectively. The interface of this two-layer model is at a depth of $0.5 \mathrm{~km}$. Shot gathers are computed for 100 shots spaced at $20 \mathrm{~m}$ intervals. Each shot is recorded using 200 receivers separated at an interval of $10 \mathrm{~m}$ on the surface. The migration velocity model is homogeneous with the velocity of $1400 \mathrm{~m} / \mathrm{s}$. Figures 7a-e show that the traces with different offset ranges are migrated to different depths, and the reflector images shift to shallower depths with increasing source-receiver offsets. Figure $7 \mathrm{f}$ shows the migration image using the full-offset data, which is quite similar to the migration image using only the near-offset data shown in Figure 7a. This similarity can be explained by the stationary phase theory (Schuster, 2009), except there are still significant artifacts due to incomplete cancellation (see Figure 7f).

Figure 8a shows the comparison between the observed traces (red wiggles) and the Born data (green wiggles) which are computed from the migration velocity model and the migration image in Figure 7f. Two phenomena can be noticed in Figures 8(a): (1) The far-offset data suffer severely from cycle-skipping and (2) the seismic events in the Born modeled data arrive earlier at the near-offset region compared to the observed data. However, the Born modeled events gradually arrive later as the offset increases. These phase mismatches can be seen more clearly in Figure 8b, which are the zoomed views of the blue boxes in Figure 8a. Moreover, the amplitudes of the Born reflections are much weaker compared to the observed data as the out-of-phase summation severely weakens the amplitudes of the migration image. This phenomenon can be observed in Figure 8c, which shows the data residuals of Figure 8a. It clearly illustrates that the data residual is dominated by the energy of the observed data. 
Figure 7a shows the migration image computed from the near-offset data with source-receiver offsets smaller than $0.2 \mathrm{~km}$. This image has fewer amplitude and phase errors compared to Figure $7 \mathrm{f}$ because the shorter wavepaths suffer less from velocity errors. The comparisons between the observed and Born data generated from Figure 7a are shown in Figure 9a. Even though there are still severe cycle-skipping problems at the far-offset region, their waveforms correlate very well at the near-offset region. This behavior is even more pronounced in the zoomed view shown in Figure 9b. Figure 9c shows the data residuals in Figure 9a, where the residual traces are almost negligible because the observed and recorded data mostly agree with one another in both their phases and amplitudes. The blue box indicates the offset region used to compute the migration image.

The rolling-offset strategy is now tested on three different cases. In the first example, we use full-offset data for both migration and inversion. Therefore, the RFWI gradient suffers from cycle skipping as well as amplitude and phase mismatches at both the near and far offsets. The corresponding RFWI gradient is shown in Figure 10a, which exhibits strong aliasing artifacts along the sides of the computational model. In the second example, we use full-offset data for migration, but we only select the uncycle-skipped data at the near-offsets for inversion. To mitigate the amplitude mismatches, we normalize both the computed Born and the observed data before subtraction. Therefore, the phase mismatch problem is the dominant one in the second case. Figure 10b shows the RFWI gradient for the second case, which has balanced low-wavenumber updates in the central part of the model. However, there is still aliasing along the side boundaries. In the third example, we use near-offset uncycle-skipped data for both migration and inversion. The corresponding RFWI gradient is shown in Figure 10c, which generates strong and balanced low-wavenumber updates. 


\section{Discussion of the Offset Selection}

To remove the cycle-skipped traces, we can either calculate their traveltime or phase differences. Cycle skipping occurs when the traveltime or phase differences between the synthetic and observed traces are larger than $\frac{T}{2}$ or $\pi$, respectively (Shah et al., 2012). Here, $T$ is the period of the seismic signal. The traveltime difference $\Delta \tau$ can be found by maximizing the crosscorrelation function $f(\tau)$ as

$$
\max \{f(\tau)\}=\max \left\{\int p\left(\mathbf{x}_{r}, t ; \mathbf{x}_{s}\right)_{o b s} p\left(\mathbf{x}_{r}, t+\tau ; \mathbf{x}_{s}\right)_{s y n} d t\right\}
$$

where $p\left(\mathbf{x}_{r}, t ; \mathbf{x}_{s}\right)_{o b s}$ represents the observed trace at the receiver location $\mathbf{x}_{r}$ for a shot at location $\mathbf{x}_{s}$. Similarly, $p\left(\mathbf{x}_{r}, t+\Delta \tau ; \mathbf{x}_{s}\right)_{s y n}$ indicates the synthetic trace at the same source and receiver location but with a time shift $\tau$. This equation seeks a $\Delta \tau$ that shifts the synthetic seismogram so that it best matches the observed seismogram.

The phase difference $\Delta \phi$ between the synthetic and observed seismic traces can be computed by two steps. First, transform the observed and synthetic traces into the frequency domain by FFT

$$
\begin{aligned}
& \tilde{p}_{o b s}=\mathscr{F}\left(p\left(\mathbf{x}_{r}, t ; \mathbf{x}_{s}\right)_{o b s}\right)=A_{o b s} e^{i \phi_{o b s}} \\
& \tilde{p}_{s y n}=\mathscr{F}\left(p\left(\mathbf{x}_{r}, t ; \mathbf{x}_{s}\right)_{s y n}\right)=A_{s y n} e^{i \phi_{s y n}}
\end{aligned}
$$

where $A_{o b s}$ and $A_{\text {syn }}$ represent the magnitude spectra of the observed and synthetic seismic traces, respectively. $\phi_{o b s}$ and $\phi_{s y n}$ indicate their corresponding phase spectrums. Second, compute their phase difference by

$$
\begin{aligned}
\Delta \phi & =\operatorname{phase}\left(\tilde{p}_{\text {obs }} \tilde{p}_{\text {syn }}^{*}\right)=\operatorname{phase}\left(A_{\text {obs }} A_{\text {syn }} e^{i\left(\phi_{o b s}-\phi_{s y n}\right)}\right) \\
& =\phi_{\text {obs }}-\phi_{\text {syn }}
\end{aligned}
$$


where $*$ is the conjugate symbol and phase indicates a phase extraction operator. In our paper, we prefer the phase difference approach rather than the traveltime difference approach. The reason is that the phase difference $\Delta \phi$ varies smoothly with increasing offset if the seismic traces are not cycle-skipped. However, a sudden $2 \pi$ phase jump will occur at the cycle-skipped trace. This phase jump is easy to detect and can be used directly to remove the cycle-skipped traces.

Figure $11 \mathrm{~b}$ shows a comparison between two shots where the nearest-offset trace is in the middle. The reflection events in the two shot gathers disagree more with increasing offset. Figure 11a shows the corresponding phase differences which clearly shows the phase-jump points. These phase-jump points indicate the locations where the cycle-skipping appears. We can simply remove the traces where its offset is larger than the phase jump locations to avoid the cycle-skipping problem.

The offset range associated with uncycle-skipped traces is easy to identify for a simple case where there is only one seismic event per trace. However, for complicated cases, our offset selection strategy is the following:

1. Migrate the observed data with the initial velocity model. Then manually pick the points of the reference reflector which is relatively continuous and has a good signal-to-noise ratio.

2. Generate the Born data based on the picked reflectivity model and window out the corresponding reflections in the observed data.

3. Measure the phase differences between the Born data and the windowed observe data, then identify the offset with the first phase jump point as a reference to remove the cycle-skipped seismic traces.

Theoretically, one can pick single or multiple reflectors as the reference to guide the offset selection procedure. The more the reflectors are used, the higher the accuracy can be achieved in removing cycle-skipped traces. 


\section{Misfit Function}

The misfit function of multiscale reflection phase inversion is defined as

$$
\begin{aligned}
\epsilon_{i} & =\sum_{s, r} \int d t\left[W_{i} M I^{n}\left(d\left(\mathbf{x}_{r}, t \mid \mathbf{x}_{s}\right)-d_{o b s}\left(\mathbf{x}_{r}, t \mid \mathbf{x}_{s}\right)\right)\right]^{2} \\
& =\sum_{s, r} \int d t\left[I^{n}\left(\bar{d}_{i}-\bar{d}_{i_{-o b s}}\right)\right]^{2}
\end{aligned}
$$

where $W_{i}$ is a weighting operator that zeros out the cycle-skipped events in the data at the $i$ th stage. Here, $M$ indicates the amplitude replacement procedure in equation $8, I^{n}$ is the integration operator $I \equiv \int d t$ and $n$ indicates that the integration is performed $n$ times. The observed data $d_{o b s}$ only contain the reflection energy, $d$ represents the synthetic data generated from the Born modeling, and $\bar{d}$ and $\bar{d}_{\text {obs }}$ represent the modified traces. The gradient of the misfit function $\epsilon$ with respect to velocity $c$ is given by

$$
\begin{aligned}
\frac{\partial \epsilon_{i}}{\partial c} & =\sum_{s, r} \int d t\left(I^{n} \frac{\partial \bar{d}_{i}}{\partial c}\right)^{T}\left(I^{n} \Delta \bar{d}_{i}\right) \\
& =-\sum_{s, r} \int_{0}^{T} 2 \rho c\left(I^{n} \nabla \cdot \mathbf{v}\right)\left(I^{n} \delta q\right)+2 \rho c\left(I^{n} \nabla \cdot \delta \mathbf{v}\right)\left(I^{n} q\right) d t
\end{aligned}
$$

where $\mathbf{v}$ and $\delta \mathbf{v}$ represent the background and perturbed particle-velocity fields, respectively, and $q$ and $\delta q$ indicate the adjoint-state variables of the background and perturbed pressure wavefield $p$ and $\delta p$, respectively. The density is represented as $\rho$. As an alternative, the integration operator can be applied to the source wavelet directly. Therefore, equation 9 can be simplified to

$$
\frac{\partial \epsilon_{i}}{\partial c}=-\sum_{s, r} \int_{0}^{T} 2 \rho c(\nabla \cdot \tilde{\mathbf{v}}) \delta \tilde{q}+2 \rho c(\nabla \cdot \delta \tilde{\mathbf{v}}) \tilde{q} d t
$$

where the tilde indicates that the wavefields are generated by an integrated source. The detailed derivation of the gradient is shown in Appendix B using the adjoint-state method. 
The gradient in equation 10 has two terms. This first term $\int_{0}^{T} 2 \rho c(\nabla \cdot \tilde{\mathbf{v}}) \delta \tilde{q} d t$ corresponds to the source-side reflection wavepath, which is the dot product between the downward-propagated source-side wavefield $\nabla \cdot \tilde{\mathbf{v}}$ and the upward-propagated receiver-side Born wavefield $\delta \tilde{q}$. Similarly, the second term $\int_{0}^{T} 2 \rho c(\nabla \cdot \delta \tilde{\mathbf{v}}) \tilde{q} d t$ can be interpreted as the receiver-side reflection wavepath, which is the temporal dot product between the downward-propagated receiver-side wavefield $\tilde{q}$ and the upward-propagated sourceside Born wavefield $\nabla \cdot \delta \tilde{\mathbf{v}}$. The background velocity is updated by smearing the energy of the data misfit along the wavepaths associated with the sources and receivers.

For a single source-receiver pair, Figure $12 \mathrm{~b}$ shows the reflection wavepath of MRPI with the integration time $n=3$, which is smoother, wider and more balanced in the spatial distribution of amplitudes compared to the reflection wavepath of conventional RFWI shown in Figure 12a. Given the gradient, the steepestdescent method can be used to iteratively update the background-velocity model until the data misfit is sufficiently small. In this paper, we only use the steepest descent (SD) method for model updates. However, more advanced method, such as Gauss-Newton, full Newton and $l$-BGFS, can be used to achieve a better gradient quality and a faster convergence. Pratt et al. (1998) proved the advantage of using the full Newton method in estimating the background velocities. Pan et al. (2017) clearly shows the advantage of $l$-BFGS method on both convergence speed and inversion quality in recovering a Gaussian-anomaly model when compared to the SD method.

\section{WorkFlow of MRPI}

The workflows for conventional RFWI and MRPI + MD method are compared in Figure 13. For the conventional RFWI workflow shown in Figure 13a, the observed data are directly fed into the migration and inversion algorithms. In this case, the convergence of conventional RFWI can be hardly guaranteed. Figure $13 \mathrm{~b}$ provides the workflow of the multiscale reflection phase inversion: 
1. The observed traces are temporally integrated $\mathrm{n}$ times to attenuate the high-frequency information and boost the low-frequency information.

2. Select the near-offset uncycle-skipped data to compute the migration image using MD.

3. Compare the Born modeled data with the observed data, select the uncycle-skipped traces again to compute the reflection wavepaths and update the background velocity model.

4. Compute the new migration image based on the updated velocity model.

5. Repeat steps 3 - 4 until the residual falls below a specified tolerance. Reduce the integration number from $n$ to $n-1$ and repeat steps 2 - 4 again. For all of the iterations, the synthetic magnitude spectra are replaced with the observed spectra.

This workflow mitigates the problem of getting stuck in a local minimum and is computationally less expensive than standard RFWI which uses many iterations for the LSM inner loop.

\section{Quantitative Analysis of MRPI + MD Method}

The MRPI + MD method is similar to the iterative block Gauss-Seidel method for solving systems of equations. The goal of iterative block Gauss-Seidel (IBGS) is to iteratively find the solution to a large system of equations. The large matrix is broken up into smaller blocks, where there is no or very little overlap between blocks and an initial starting model is specified. The solution associated with the top block of equations is found first. This solution is then used as the starting solution for the next block of equations. This procedure is repeated until the solution to the last block of equations is found. However, the equations for the MRPI procedure are non-linear with respect to the model parameters, so that we use a non-linear IBGS procedure where the coefficients in the matrix blocks are updated after a sufficient number of sweeps through the entire matrix. 
The equations for MRPI + MD can be arranged from top to bottom in blocks of traces with different offset ranges (for simplicity, we use the linearized equations for demonstration and also ignore the integration times)

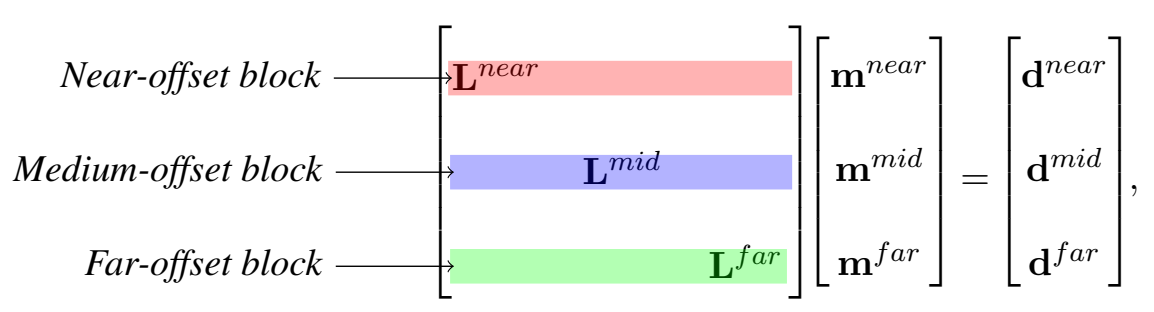

where $\mathbf{L}^{\text {near }}, \mathbf{L}^{\text {mid }}$ and $\mathbf{L}^{\text {far }}$ represent the block of modeling operators, each with a different offset-range. Each modeling operator can be mathematically represented as $W G(\mathbf{r} \mid \mathbf{x}) G(\mathbf{x} \mid \mathbf{s})$. Here, $W, G(\mathbf{x} \mid \mathbf{s})$ and $G(\mathbf{r} \mid \mathbf{x})$ indicate the source wavelet, the Green's function from the source location $\mathbf{s}$ to the pertubation point $\mathbf{x}$ and from the pertubation point $\mathbf{x}$ to the receiver at $\mathbf{r}$, respectively. Here, $\mathbf{m}$ and $\mathbf{d}$ represent the corresponding model parameters and seismic traces with different offset ranges.

In equation 11, the solution of each block is found by the procedure listed in the MRPI + MD workflow. Once the model $\mathbf{m}^{\text {near }}$ is founded that explains the near-offset traces $\mathbf{d}^{\text {near }}$, then the model $\mathbf{m}^{\text {near }}$ is used as the starting model for the next block of traces. This procedure is repeated until the last block of equations is solved. In the practical case, to ensure that the inverted model from the later blocks is still able to explain the seismic traces in the previous block, we solve the system of equations in the following way:

1. Solve the first block in equation 11 to invert for $\mathbf{m}^{\text {near }}$.

2. Use $\mathbf{m}^{\text {near }}$ as the starting model to solve the first block in equation 12

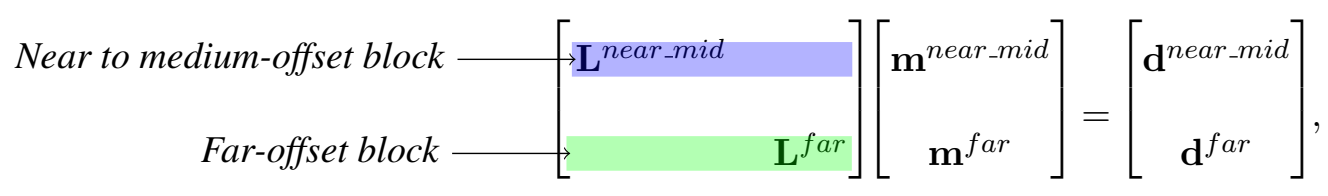


where the near- to medium-offset block indicated by the blue color is the combination of the near- and medium- block in equation 11 . The inverted model $\mathbf{m}^{\text {near_mid }}$ is mostly inverted from the medium offset data $\mathbf{d}^{\text {mid }}$, while still satisfying the system of equations in the first block of equation 11.

3. Use $\mathbf{m}^{\text {near_mid }}$ as the starting model to solve equation

$$
\text { Full-offset block } \longrightarrow\left[\mathbf{L}^{\text {near_mid_far }}\right]\left[\mathbf{m}^{\text {near_mid_far }}\right]=\left[\mathbf{d}^{\text {near_mid_far }}\right],
$$

Similar to the IGBS method, this block-by-block method is repeated until convergence.

\title{
NUMERICAL RESULTS
}

\begin{abstract}
We now use synthetic data and recorded marine data to test the effectiveness and advantages of MRPI + MD compared to conventional FWI and RFWI. The marine data are field OBC traces recorded in a North Sea seismic survey. In all of the examples, the first arrivals are muted from the traces to ensure that the velocity updates are only contributed by the reflection wavepaths.
\end{abstract}

\section{Two-Layer Model}

We first use a two-layer model to illustrate the merits of MRPI + MD compared to conventional FWI and RFWI. Figure 15a depicts the velocity model used to generate the data. There are 100 shots evenly spaced at a distance of $20 \mathrm{~m}$ and each shot is recorded using 200 receivers placed on the surface with an interval of $10 \mathrm{~m}$. The source function is a Ricker wavelet with a peak frequency of $25 \mathrm{~Hz}$. A time-domain first-order acoustic finite-difference modeling algorithm is used for both data simulations and inversion. The initial velocity model is homogeneous with $v=1400 \mathrm{~m} / \mathrm{s}$, which is about $23 \%$ smaller than the true velocity.

Figures $14 \mathrm{a}$ and $15 \mathrm{c}$ show, respectively, the virtual source and the corresponding gradient at the 1 st 
iteration of conventional FWI. This gradient is dominated by high-wavenumber energy where the lowwavenumber information is slightly updated. Figure $14 \mathrm{~b}$ displays the virtual source from conventional RFWI which suffers from severe cycle-skipping problems. Therefore, the gradient of conventional RFWI in Figure 15e contains strong aliasing artifacts which promote incorrect updates of the velocity model. The virtual source from the MRPI + MD method is shown in Figure 14c where the cycle-skipped data are muted out. Figure $15 \mathrm{~g}$ shows the MRPI + MD gradient which is characterized by balanced amplitudes at lowwavenumbers. The final tomograms computed from these methods are shown in Figures $15 \mathrm{~d}, 15 \mathrm{f}$, and $15 \mathrm{~h}$, where both FWI and RFWI fail to update the velocity model.

\section{Marmousi Model}

Data computed from the Marmousi model are used to test the MRPI + MD method. We select the upperright region of the Marmousi model with a size of 121 x 272 grid points. We then extend the model by 50 grid points on each side. The finite-difference method is used to compute 186 shot gathers on the surface of the Figure 16a model with $20 \mathrm{~m}$ shot intervals. Each shot is recorded with 800 receivers that are evenly distributed on the surface at a spacing of $10 \mathrm{~m}$. A Ricker wavelet with a peak frequency of $15 \mathrm{~Hz}$ is used as the source wavelet and the initial velocity model is shown in Figure $16 \mathrm{~b}$.

Figures $16 \mathrm{c}$ to $16 \mathrm{~g}$ compares the gradients at the first iteration for the FWI, RFWI and MRPI + MD methods. The FWI gradient in Figure 16c is dominated by high-wavenumber components, where the lowwavenumber information is hardly updated. Figures $16 \mathrm{e}$ and $16 \mathrm{~g}$ display the gradients for RFWI and MRPI + MD, which are mainly controlled by the low-wavenumber components. However, the RFWI gradient fails to update the right part of the model. The final inverted models are compared in Figures 16d-h, where the FWI tomogram in Figure 16b mainly updates the high-wavenumber components of the velocity model. The RFWI tomogram shown in Figure $16 \mathrm{f}$ mostly updates the low-wavenumber components in the shallow area 
and largely ignores the deeper portions of the velocity model. The weak updates in the deeper parts of the velocity model are largely a result of cycle-skipping between the observed and predicted traces. Figure $16 \mathrm{~h}$ displays the tomogram inverted by MRPI + MD, where the low-wavenumber components of the velocity model are largely recovered. Figure 17 compares the vertical profiles between the inverted results and the true model, which suggests that MRPI + MD is more accurate than the conventional waveform inversion method.

The migration images computed from the tomograms in Figure 16 are shown in Figure 18. Here, the FWI and RFWI migration images fail to accurately image the deep reflectors. In comparison, the deep reflectors are accurately imaged by the MRPI + MD tomogram with more balanced amplitudes and higher resolution.

\section{Volve OBC Data}

We test the effectiveness of MRPI + MD using a 2D slice of inline shot gathers extracted from the 3D Volve OBC data (Fu et al., 2017). The 3D dataset consists of 12 parallel cables with $400 \mathrm{~m}$ separations, with each containing 240 receivers. The 2D dataset is extracted from line 6 (calbe 6 ) which is in the middle of the survey. The recorded data consist of 240 common-shot gathers with a shot interval of around $50 \mathrm{~m}$, and the number of receivers for each shot ranges from 77 to 237 with a receiver interval of $50 \mathrm{~m}$. The data were recorded with a sampling interval of $4 \mathrm{~ms}$ for the total recording time of $7.504 \mathrm{~s}$. The lowest available frequency of the data is about $2 \mathrm{~Hz}$. During processing, the data are low-pass filtered to $14 \mathrm{~Hz}$ and resampled to $1 \mathrm{~ms}$. The traces are muted after $5 \mathrm{~s}$ as there are no reflections below $5 \mathrm{~s}$. Figures 19a and $19 \mathrm{~b}$ show the comparison between raw and processed shot gathers, where the reflections arriving before 2 s have a relatively low signal-to-noise (SNR) ratio; therefore, these low SNR reflections are also removed. We also apply a time gain to compensate for the 3D effects. Finally, the traces are normalized to enhance the 
contribution of the far-offset traces and the source time history is a Ricker wavelet with the peak frequency of $7 \mathrm{~Hz}$. We do not estimate the source from the data because the migrated image automatically contains the source signature information embedded in the observed data (Wang et al., 2013). Figures 19b-c compare the original processed data before and after three integrations. Their normalized magnitude spectra are shown in Figure 19d where the low-frequency components in the data are largely boosted from the integration.

The arrivals between 2.5-3.0 s are the reflections from the chalk layer (Duan et al., 2017). Applying FWI, RFWI and MRPI + MD to these data result in the inverted tomograms shown in Figure 20. The data are separated into four offset groups for migration and inversion, which are 1250/1875 m, 1875/2650 $\mathrm{m}, 2650 / 3250 \mathrm{~m}$ and $3250 / 5000 \mathrm{~m}$. The first and second number in each group indicates the offset range of data for migration and inversion, respectively. Here the data are separated into the four different offset groups by following the workflow described in the previous "Discussion of the Offset Selection" section. The chalk layer located at the depth around $2.5 \mathrm{~km}$ is selected as the reference reflector and equation 8 is used to decide the optimal data offset under the current velocity model. Once the offset is selected, we update the velocity model with the traces in this offset range. The velocity is updated until the residual decrease fall below a specified threshold. We repeat this procedure until the end of the offset range is reached. The migration images computed from the different velocity models in Figure 20 are shown in Figure 21. Figure 21d is the migration image calculated from the MRPI + MD tomogram, which has a wider imaging area and the reflectors are more focused and continuous when compared to the others. The red arrows point to the areas in which noticeable improvements in image quality can be seen.

To further test for the accuracy of the tomograms, the sub-offset gathers shown in Figures 22a-b are computed from the initial velocity model and MRPI tomograms. The energy in the sub-offset gather in Figure 22a is spread out over a large range of sub-offset values, which indicates that the initial model is far away from the true model. But the energy of the sub-offset gather in Figure $22 b$ is mostly focused at zero sub-offset, which indicates that the MRPI + MD tomogram is a good representation of the true velocity 
model. Figures 23a-b show the angle-domain common image gathers (ADCIGs) calculated from the initial model and MRPI + MD tomogram, respectively. It is obvious that the ADCIGs associated with the MRPI + MD tomogram is more flattened than those from the initial model. The observed and Born data from the MRPI + MD tomogram are compared in Figure 24. The red and green wiggles represent the observed and Born data, respectively, where their phases are in good agreement.

\section{CONCLUSIONS}

We combine multiscale reflection phase inversion (MRPI) with migration deconvolution (MD) for inverting for the low-wavenumber components of the velocity model. This method can recover the low-wavenumber components of the velocity model more efficiently and is less prone to getting stuck in a local minima compared to the conventional RFWI method. To reduce computation costs, migration deconvolution is used as an inexpensive alternative to least-squares migration to efficiently accelerate the convergence rate. To alleviate cycle-skipping, the low-frequency information in the traces is boosted by temporally integrating the traces several times. The rolling-offset strategy is used to eliminate the cycle-skipping traces before migration and inversion. These two strategies largely reduce the tendency of MRPI getting stuck in a localminimum. The amplitude replacement procedure eliminates the need to explain the magnitude spectra in the recorded traces, so that phase mismatches are mostly related to the kinematic errors that result from an inaccurate velocity model. Tests with both synthetic and real data demonstrate the effectiveness of MRPI + MD in efficiently recovering the low-wavenumber portions of the velocity model with acceptable accuracy.

\section{ACKNOWLEDGMENTS}

The research reported in this paper was supported by the King Abdullah University of Science and Technology (KAUST) in Thuwal, Saudi Arabia. We are grateful to the sponsors of the Center for Subsurface 
Imaging and Modeling (CSIM) Consortium for their financial support. For computer time, this research used the resources of the Supercomputing Laboratory at KAUST. We thank them for providing the computational resources required for carrying out this work. The authors would like to thank Statoil ASA and the Volve license partners ExxonMobil E\&P Norway AS and Bayerngas Norge AS, for the release of the Volve data. The views expressed in this paper are the views of the authors and do not necessarily reflect the views of Statoil ASA and the Volve field license partners.

\section{APPENDIX A}

\section{TIME INTEGRATION AS A LOW-FREQUENCY BOOST FILTER}

Assume a time-domain signal $g(t)$ is equal to $G(f)$ in the frequency domain. According to the derivative property of the Fourier transform, we have

$$
F\left(\frac{d g(t)}{d t}\right)=i 2 \pi f G(f)
$$

where $F$ represents the Fourier transform operator. We can re-write equation A-1 as

$$
F\left(\frac{d g(t)}{d t}\right)=i 2 \pi f F(g(t))
$$

and substitute $p(t)=\frac{d g(t)}{d t}$ into equation A-2 to get

$$
F(p(t))=i 2 \pi f F\left(\int_{-\infty}^{t} p(\tau) d \tau\right) .
$$


Dividing $i 2 \pi f$ on both side of the equation gives

$$
F\left(\int_{-\infty}^{t} p(\tau) d \tau\right)=\frac{F(p(t))}{i 2 \pi f}
$$

We conclude that integrating a signal in the time domain is equal to divide by $i 2 \pi f$ in the frequency domain. In this case, high-frequency information will be attenuated and the low-frequency information will become dominant. However, if there is a DC component $b$ in the signal $g(t)$ as $g(t)+b$, then the equation $p(t)=\frac{d(g(t)+b)}{d t}$ is also true. The value of the DC term increases quickly with an increase in the number of integrations. This DC term can be simply removed by demeaning the signal prior to inversion.

\section{APPENDIX B}

\section{IMAGING CONDITION OF REFLECTION INVERSION}

The first-order acoustic wave equation is

$$
\left[\begin{array}{cc}
\frac{\partial}{\partial t} & \rho c^{2} \nabla \\
\frac{1}{\rho} \nabla & \frac{\partial}{\partial t}
\end{array}\right]\left[\begin{array}{l}
P \\
\mathbf{v}
\end{array}\right]=\left[\begin{array}{l}
F \\
0
\end{array}\right]
$$

where $P$ and $\mathbf{v}$ represent the background pressure wavefield and particle-velocity field, respectively, $\rho$ is the density, $c$ indicates the velocity and the source term is represented by $F$. This equation can be rewritten more compactly as

$$
\mathbf{S}\left(\mathbf{m}_{\mathbf{s}}\right) \mathbf{w}\left(\mathbf{m}_{\mathbf{s}}\right)=\mathbf{F},
$$

where

$$
\mathbf{S}=\left[\begin{array}{cc}
\frac{\partial}{\partial t} & \rho c^{2} \nabla \\
\frac{1}{\rho} \nabla & \frac{\partial}{\partial t}
\end{array}\right], \mathbf{w}=\left[\begin{array}{l}
P \\
\mathbf{v}
\end{array}\right], \mathbf{F}=\left[\begin{array}{l}
F \\
0
\end{array}\right] .
$$


Here, $\mathbf{w}$ represent the background wavefield and $\mathbf{S}$ indicates as the forward modeling operator. The model parameter is defind as $\mathbf{m}=\left(\begin{array}{l}\mathbf{m}_{\mathbf{s}} \\ \mathbf{m}_{\mathbf{r}}\end{array}\right)$, where $\mathbf{m}_{\mathbf{s}}$ is the smooth background model and $\mathbf{m}_{\mathbf{r}}$ is the reflectivity model. Similarly, the Born modeling equation can be written as

$$
\mathbf{S}\left(\mathbf{m}_{\mathbf{s}}\right) \delta \mathbf{w}(\mathbf{m})=\delta \mathbf{F}\left(\mathbf{w}, \mathbf{m}_{\mathbf{r}}\right)
$$

where $\delta \mathbf{w}$ represents the perturbed wavefield and $\delta \mathbf{F}$ is the virtual source calculated from the background wavefield $\mathbf{w}$ and the reflectivity model $\mathbf{m}_{\mathbf{r}}$.

The least-squares misfit function $\epsilon(\mathbf{m})$ for the model parameter $\mathbf{m}$ can be written as

$$
\epsilon(\mathbf{m})=\frac{1}{2}\langle\delta \mathbf{w}(\mathbf{m})-\mathbf{d}, \delta \mathbf{w}(\mathbf{m})-\mathbf{d}\rangle
$$

where $\delta \mathbf{w}(\mathbf{m})$ and $\mathbf{d}$ represent the predicted and recorded data, respectively. The gradient of $\epsilon(\mathbf{m})$ with respect to the model parameter $\mathbf{m}$ is given by

$$
\frac{\partial \epsilon(\mathbf{m})}{\partial \mathbf{m}}=\left\langle\frac{\partial \delta \mathbf{w}}{\partial \mathbf{m}}, \Delta \mathbf{d}\right\rangle
$$

Computing the derivative of equation B-4 with respect to the model parameter $\mathbf{m}$, we get

$$
\begin{aligned}
& \frac{\partial \mathbf{S}\left(\mathbf{m}_{\mathbf{s}}\right)}{\partial \mathbf{m}} \delta \mathbf{w}(\mathbf{m})+\mathbf{S}\left(\mathbf{m}_{\mathbf{s}}\right) \frac{\partial \delta \mathbf{w}(\mathbf{m})}{\partial \mathbf{m}}=\frac{\delta \mathbf{F}\left(\mathbf{w}\left(\mathbf{m}_{\mathbf{s}}\right), \mathbf{m}_{\mathbf{r}}\right)}{\partial \mathbf{m}} \\
\Rightarrow & \frac{\partial \delta \mathbf{w}(\mathbf{m})}{\partial \mathbf{m}}=\mathbf{S}^{-1}\left(\frac{\delta \mathbf{F}\left(\mathbf{w}\left(\mathbf{m}_{\mathbf{s}}\right), \mathbf{m}_{\mathbf{r}}\right)}{\partial \mathbf{m}}-\frac{\partial \mathbf{S}\left(\mathbf{m}_{\mathbf{s}}\right)}{\partial \mathbf{m}} \delta \mathbf{w}(\mathbf{m})\right)
\end{aligned}
$$

where

$$
\frac{\delta \mathbf{F}\left(\mathbf{w}\left(\mathbf{m}_{\mathbf{s}}\right), \mathbf{m}_{\mathbf{r}}\right)}{\partial \mathbf{m}}=\frac{\partial \delta \mathbf{F}\left(\mathbf{w}\left(\mathbf{m}_{\mathbf{s}}\right), \mathbf{m}_{\mathbf{r}}\right)}{\partial \mathbf{w}\left(\mathbf{m}_{\mathbf{s}}\right)} \frac{\partial \mathbf{w}\left(\mathbf{m}_{\mathbf{s}}\right)}{\partial \mathbf{m}}+\frac{\partial \delta \mathbf{F}\left(\mathbf{w}\left(\mathbf{m}_{\mathbf{s}}\right), \mathbf{m}_{\mathbf{r}}\right)}{\partial \mathbf{m}_{\mathbf{r}}} \frac{\partial \mathbf{m}_{\mathbf{r}}}{\partial \mathbf{m}}
$$


Note that $\mathbf{m}=\left(\mathbf{m}_{\mathbf{s}}, \mathbf{m}_{\mathbf{r}}\right)$, so that

$$
\frac{\partial \mathbf{m}_{\mathbf{r}}}{\partial \mathbf{m}}=\left\{\begin{array}{ll}
1, & \text { if } \mathbf{m}=\mathbf{m}_{\mathbf{r}} \\
0, & \text { if } \mathbf{m}=\mathbf{m}_{\mathbf{s}}
\end{array}, \quad \frac{\partial \mathbf{w}\left(\mathbf{m}_{\mathbf{s}}\right)}{\partial \mathbf{m}}= \begin{cases}\frac{\partial \mathbf{w}\left(\mathbf{m}_{\mathbf{s}}\right)}{\partial \mathbf{m}}, & \text { if } \mathbf{m}=\mathbf{m}_{\mathbf{s}} \\
0, & \text { if } \mathbf{m}=\mathbf{m}_{\mathbf{r}}\end{cases}\right.
$$

Similarly, taking the derivative of equation B-2 with respect to the model parameter $\mathbf{m}$, we have

$$
\frac{\partial \mathbf{w}\left(\mathbf{m}_{\mathbf{s}}\right)}{\partial \mathbf{m}}=-\mathbf{S}\left(\mathbf{m}_{\mathbf{s}}\right)^{-1} \frac{\partial \mathbf{S}\left(\mathbf{m}_{\mathbf{s}}\right)}{\partial \mathbf{m}} \mathbf{w}
$$

Inserting Equation B-7, B-8 and B-10 into Equation B-6, gives

$$
\begin{aligned}
\frac{\epsilon(\mathbf{m})}{\partial \mathbf{m}} & =\left\langle\frac{\partial \delta \mathbf{F}\left(\mathbf{w}\left(\mathbf{m}_{\mathbf{s}}\right), \mathbf{m}_{\mathbf{r}}\right)}{\partial \mathbf{w}\left(\mathbf{m}_{\mathbf{s}}\right)}\left(-\mathbf{S}\left(\mathbf{m}_{\mathbf{s}}\right)^{-1}\right) \frac{\partial \mathbf{S}\left(\mathbf{m}_{\mathbf{s}}\right)}{\partial \mathbf{m}} \mathbf{w}+\frac{\partial \delta \mathbf{F}\left(\mathbf{w}\left(\mathbf{m}_{\mathbf{s}}\right), \mathbf{m}_{\mathbf{r}}\right)}{\partial \mathbf{m}_{\mathbf{r}}} \frac{\partial \mathbf{m}_{\mathbf{r}}}{\partial \mathbf{m}}-\frac{\partial \mathbf{S}\left(\mathbf{m}_{\mathbf{s}}\right)}{\partial \mathbf{m}} \delta \mathbf{w}(\mathbf{m}),\left(\mathbf{S}\left(\mathbf{m}_{\mathbf{s}}\right)^{-1}\right)^{*} \Delta \mathbf{d}\right\rangle \\
& =-\left\langle\frac{\partial \delta \mathbf{F}\left(\mathbf{w}\left(\mathbf{m}_{\mathbf{s}}\right), \mathbf{m}_{\mathbf{r}}\right)}{\partial \mathbf{w}\left(\mathbf{m}_{\mathbf{s}}\right)} \mathbf{S}\left(\mathbf{m}_{\mathbf{s}}\right)^{-1} \frac{\partial \mathbf{S}\left(\mathbf{m}_{\mathbf{s}}\right)}{\partial \mathbf{m}} \mathbf{w}, \mathbf{w}^{*}\right\rangle-\left\langle\frac{\partial \mathbf{S}\left(\mathbf{m}_{\mathbf{s}}\right)}{\partial \mathbf{m}} \delta \mathbf{w}(\mathbf{m}), \mathbf{w}^{*}\right\rangle+\left\langle\frac{\partial \delta \mathbf{F}\left(\mathbf{w}\left(\mathbf{m}_{\mathbf{s}}\right), \mathbf{m}_{\mathbf{r}}\right)}{\partial \mathbf{m}_{\mathbf{r}}} \frac{\partial \mathbf{m}_{\mathbf{r}}}{\partial \mathbf{m}}, \mathbf{w}^{*}\right\rangle
\end{aligned}
$$

where $*$ indicates the adjoint. Here, we denote $\mathbf{w}(\mathbf{m})^{*}=\left(\mathbf{S}\left(\mathbf{m}_{\mathbf{S}}\right)^{-1}\right)^{*} \Delta \mathbf{d}$ as the solution of the adjoint equation with the residual resismograms acting as virtual soures:

$$
\mathbf{S}^{*}\left(\mathbf{m}_{\mathbf{s}}\right) \mathbf{w}^{*}(\mathbf{m})=\Delta \mathbf{d}
$$

where $\mathbf{S}^{*}$ is the adjoint operator of $\mathbf{S}$. Therefore, according to equation B-12, the gradient with respect to 
the background model $\mathbf{m}_{\mathbf{s}}$ can be written as

$$
\begin{aligned}
\frac{\epsilon(\mathbf{m})}{\partial \mathbf{m}_{\mathbf{s}}} & =-\left\langle\frac{\partial \delta \mathbf{F}\left(\mathbf{w}\left(\mathbf{m}_{\mathbf{s}}\right), \mathbf{m}_{\mathbf{r}}\right)}{\partial \mathbf{w}\left(\mathbf{m}_{\mathbf{s}}\right)} \mathbf{S}\left(\mathbf{m}_{\mathbf{s}}\right)^{-1} \frac{\partial \mathbf{S}\left(\mathbf{m}_{\mathbf{s}}\right)}{\partial \mathbf{m}_{\mathbf{s}}} \mathbf{w}, \mathbf{w}^{*}\right\rangle-\left\langle\frac{\partial \mathbf{S}\left(\mathbf{m}_{\mathbf{s}}\right)}{\partial \mathbf{m}_{\mathbf{s}}} \delta \mathbf{w}(\mathbf{m}), \mathbf{w}^{*}\right\rangle \\
& =-\left\langle\frac{\partial \mathbf{S}\left(\mathbf{m}_{\mathbf{s}}\right)}{\partial \mathbf{m}_{\mathbf{s}}} \mathbf{w},\left(\mathbf{S}\left(\mathbf{m}_{\mathbf{s}}\right)^{-1}\right)^{*}\left(\frac{\partial \delta \mathbf{F}\left(\mathbf{w}\left(\mathbf{m}_{\mathbf{s}}\right), \mathbf{m}_{\mathbf{r}}\right)}{\partial \mathbf{w}\left(\mathbf{m}_{\mathbf{s}}\right)}\right)^{*} \mathbf{w}^{*}\right\rangle-\left\langle\frac{\partial \mathbf{S}\left(\mathbf{m}_{\mathbf{s}}\right)}{\partial \mathbf{m}_{\mathbf{s}}} \delta \mathbf{w}(\mathbf{m}), \mathbf{w}^{*}\right\rangle, \\
& =-\left\langle\frac{\partial \mathbf{S}}{\partial \mathbf{m}_{\mathbf{s}}} \mathbf{w}, \delta \mathbf{w}^{*}\right\rangle-\left\langle\frac{\partial \mathbf{S}}{\partial \mathbf{m}_{\mathbf{s}}} \delta \mathbf{w}, \mathbf{w}^{*}\right\rangle,
\end{aligned}
$$

where we denote $\delta \mathbf{w}^{*}=\left(\mathbf{S}\left(\mathbf{m}_{\mathbf{s}}\right)^{-1}\right)^{*}\left(\frac{\partial \delta \mathbf{F}\left(\mathbf{w}\left(\mathbf{m}_{\mathbf{s}}\right), \mathbf{m}_{\mathbf{r}}\right)}{\partial \mathbf{w}\left(\mathbf{m}_{\mathbf{s}}\right)}\right)^{*} \mathbf{w}^{*}$ as the solution of the adjoint Born equation

$$
\mathbf{S}^{*}\left(\mathbf{m}_{\mathbf{s}}\right) \delta \mathbf{w}^{*}(\mathbf{m})=\delta \mathbf{F}^{*}\left(\mathbf{w}^{*}, \mathbf{m}_{\mathbf{r}}\right)
$$

and $\mathrm{w}^{*}$ is background adjoint wavefield which satisfies the adjoint equation

$$
\mathbf{S}^{*}\left(\mathbf{m}_{\mathbf{s}}\right) \mathbf{w}^{*}(\mathbf{m})=\mathbf{F}^{*}
$$

Here,

$$
\mathbf{S}^{*}=\left[\begin{array}{cc}
-\frac{\partial}{\partial t} & -\nabla \cdot \frac{1}{\rho} \\
-\nabla \rho c^{2} & -\frac{\partial}{\partial t}
\end{array}\right], \mathbf{w}^{*}=\left[\begin{array}{l}
q \\
\mathbf{u}
\end{array}\right], \mathbf{F}^{*}=\left[\begin{array}{c}
\mathbf{\Delta} \mathbf{d} \\
0
\end{array}\right],
$$

where $q$ is the adjoint state variable of the pressure wavefield $P$ and $\mathbf{u}$ is the adjoint of the particle velocity 
vector $\mathbf{v}$. For $\mathbf{m}_{\mathbf{s}}=v$, the gradient in equation B-13 can be written as

$$
\begin{aligned}
-\left\langle\frac{\partial \mathbf{S}}{\partial \mathbf{m}_{\mathbf{s}}} \mathbf{w}, \delta \mathbf{w}^{*}\right\rangle & =-\left\langle\left[\begin{array}{cc}
0 & 2 \rho c \nabla \cdot \\
0 & 0
\end{array}\right]\left[\begin{array}{l}
P \\
\mathbf{v}
\end{array}\right],\left[\begin{array}{l}
\delta q \\
\delta \mathbf{u}
\end{array}\right]\right\rangle, \\
& =-\int_{0}^{T} 2 \rho c(\nabla \cdot \mathbf{v}) \delta q d t \\
-\left\langle\frac{\partial \mathbf{S}}{\partial \mathbf{m}_{\mathbf{s}}} \delta \mathbf{w}, \mathbf{w}^{*}\right\rangle & =-\left\langle\left[\begin{array}{cc}
0 & 2 \rho c \nabla \cdot \\
0 & 0
\end{array}\right]\left[\begin{array}{l}
\delta P \\
\delta \mathbf{v},
\end{array}\right],\left[\begin{array}{l}
q \\
\mathbf{u}
\end{array}\right]\right\rangle \\
& =-\int_{0}^{T} 2 \rho c(\nabla \cdot \delta \mathbf{v}) q d t
\end{aligned}
$$




\section{REFERENCES}

Agudo, Ò. C., N. V. da Silva, M. Warner, and J. Morgan, 2018, Acoustic full-waveform inversion in an elastic world: Geophysics. 83, no. 3, R257-R271.

AlTheyab, A. and G. Schuster, 2015, Reflection full-waveform inversion for inaccurate starting models: 2015 Workshop: Depth Model Building: Full-waveform Inversion, Beijing, China, 18-19 June 2015, $18-22$.

Aoki, N. and G. T. Schuster, 2009, Fast least-squares migration with a deblurring filter: Geophysics, 74, no. 6, WCA83-WCA93.

Asnaashari, A., R. Brossier, C. Castellanos, B. Dupuy, V. Etienne, Y. Gholami, G. Hu, L. Métivier, S. Operto, and D. Pageot, 2012, Hierarchical approach of seismic full waveform inversion: Numerical Analysis and Applications, 5, 99-108.

Bi, H. and T. Lin, 2014, Impact of adaptive data selection on full waveform inversion: 84th Annual International Meeting, SEG, Expanded Abstract, 1094-1098.

Bozdağ, E., J. Trampert, and J. Tromp, 2011, Misfit functions for full waveform inversion based on instantaneous phase and envelope measurements: Geophysical Journal International, 185, 845-870.

Brossier, R., S. Operto, and J. Virieux, 2015, Velocity model building from seismic reflection data by fullwaveform inversion: Geophysical Prospecting, 63, 354-367.

Bunks, C., F. M. Saleck, S. Zaleski, and G. Chavent, 1995, Multiscale seismic waveform inversion: Geophysics, 60, no. 5, 1457-1473.

Chen, G.-X., R.-S. Wu, and S.-C. Chen, 2018, Reflection multi-scale envelope inversion: Geophysical Prospecting, 66, 1258-1271.

Chen, Y., G. Dutta, W. Dai, and G. T. Schuster, 2017, Q-least-squares reverse time migration with viscoacoustic deblurring filters: Geophysics, 82, no. 6, S425-S438.

Chi, B., L. Dong, and Y. Liu, 2015, Correlation-based reflection full-waveform inversion: Geophysics, 80, 
no. 4, R189-R202.

Duan, Y., A. Guitton, and P. Sava, 2017, Elastic least-squares reverse time migration: Geophysics, 82, no. 4, S315-S325.

Feng, S. and G. T. Schuster, 2019, Transmission+ reflection anisotropic wave-equation traveltime and waveform inversion: Geophysical Prospecting, 67, 423-442.

Feng, Z., B. Guo, and G. T. Schuster, 2018, Multiparameter deblurring filter and its application to elastic migration and inversion: Geophysics, 83, no. 5, S421-S435.

Fu, L., B. Guo, Y. Sun, and G. T. Schuster, 2017, Multiscale phase inversion of seismic data: Geophysics, 83, no. 2, R159-R171.

Fu, L. and W. W. Symes, 2017, An adaptive multiscale algorithm for efficient extended waveform inversion: Geophysics, 82, no. 3, R183-R197.

Guitton, A., 2004, Amplitude and kinematic corrections of migrated images for nonunitary imaging operators: Geophysics, 69, no. 4, 1017-1024.

Guo, Q. and T. Alkhalifah, 2017, Elastic reflection-based waveform inversion with a nonlinear approach: Geophysics, 82, no. 6, R309-R321.

Hu, J. and G. T. Schuster, 1998, Migration deconvolution: SPIE's International Symposium on Optical Science, Engineering, and Instrumentation, 118-124.

Hu, J., G. T. Schuster, and P. A. Valasek, 2001, Poststack migration deconvolution: Geophysics, 66, no. 3, 939-952.

Lailly, P. and J. Bednar, 1983, The seismic inverse problem as a sequence of before stack migrations: Conference on inverse scattering: theory and application, SIAM, 206-220.

Pan, W., Y. Geng, and K. A. Innanen, 2018a, Interparameter trade-off quantification and reduction in isotropic-elastic full-waveform inversion: synthetic experiments and Hussar land data set application: Geophysical Journal International, 213, 1305-1333. 
Pan, W., K. A. Innanen, and Y. Geng, 2018b, Elastic full-waveform inversion and parametrization analysis applied to walk-away vertical seismic profile data for unconventional (heavy oil) reservoir characterization: Geophysical Journal International, 213, 1934-1968.

Pan, W., K. A. Innanen, and W. Liao, 2017, Accelerating Hessian-free Gauss-Newton full-waveform inversion via 1-BFGS preconditioned conjugate-gradient algorithm: Geophysics, 82, no. 2, R49-R64.

Pratt, R. G., C. Shin, and G. Hick, 1998, Gauss-Newton and full Newton methods in frequency-space seismic waveform inversion: Geophysical Journal International, 133, 341-362.

Schuster, G., 2009, Seismic Interferometry: Cambridge University Press.

Shah, N., M. Warner, T. Nangoo, A. Umpleby, I. Stekl, J. Morgan, and L. Guasch, 2012, Quality assured full-waveform inversion: Ensuring starting model adequacy: 82th Annual International Meeting, SEG, Expanded Abstracts, 1-5.

Sun, Y. and G. T. Schuster, 1993, Time-domain phase inversion: 63th Annual International Meeting, SEG, Expanded Abstracts, 684-687.

Tarantola, A., 1984, Inversion of seismic reflection data in the acoustic approximation: Geophysics, 49, $1259-1266$.

Tavares, S., 1966, A comparison of integration and low-pass filtering: IEEE Transactions on Instrumentation and Measurement, 15, 33-38.

Virieux, J. and S. Operto, 2009, An overview of full-waveform inversion in exploration geophysics: Geophysics, 74, no. 6 , WCC1-WCC26.

Wang, G., S. Wang, Q. Du, and S. Yuan, 2017, Traveltime-based reflection full-waveform inversion for elastic medium: Journal of Applied Geophysics, 141, 68-76.

Wang, S., F. Chen, H. Zhang, and Y. Shen, 2013, Reflection-based full waveform inversion (rfwi) in the frequency domain: 83th Annual International Meeting, SEG, Expanded Abstract, 877-881.

Wu, R.-S., J. Luo, and B. Wu, 2014, Seismic envelope inversion and modulation signal model: Geophysics, 
79, no. 3, WA13-WA24.

Xu, S., D. Wang, F. Chen, Y. Zhang, and G. Lambare, 2012, Full waveform inversion for reflected seismic data: 74th EAGE Conference and Exhibition, W024.

Yu, J., J. Hu, G. T. Schuster, and R. Estill, 2006, Prestack migration deconvolution: Geophysics, 71, no. 2, S53-S62. 


\section{LIST OF FIGURES}

1 The (a) true reflectivity model, (b) true velocity model, (c) reverse time migration (RTM) image, (e) least-squares reverse time migration (LSRTM) image and (g) migration deconvolution (MD) image. The RFWI gradients based on the (d) RTM, (f) LSRTM, and (h) MD images.

2 The (a) acoustic, (b) viscoacoustic and (c) elastic data. The acoustic data replaced with (d) acoustic, (e) viscoacoustic and (f) elastic magnitude spectra, respectively.

3 The data residual between the acoustic data and visco data (a) before and (b) after magnitude spectra replacement. The data residual between the acoustic data and elastic data (c) before and (d) after magnitude spectra replacement.

4 The original trace (a) without integration, (b) with one and (c) two integration.

5 The frequency spectrum of the original trace (a) without integration, with (b) one and (c) two integrations, which are represented by the red, blue and black curves, respectively.

6 Two-layer velocity model.

$7 \quad$ Migration image with offset ranges of (a) less than $0.2 \mathrm{~km}$, (b) 0.3 to $0.5 \mathrm{~km}$, (c) 0.6 to $0.8 \mathrm{~km}$, (d) 0.9 to $1.1 \mathrm{~km}$, (e) 1.2 to $1.5 \mathrm{~km}$ and (f) the entire offset.

8 The (a) trace comparsions between the observed data and Born data at $\mathrm{x}=1.0 \mathrm{~km}$; (b) zoomed view of the blue box and (c) the data residuals.

9 The (a) trace comparsion between the observed data and Born data at $\mathrm{x}=1.0 \mathrm{~km}$; (b) zoomed view of the blue box and (c) the data residuals.

10 Demonstration of the influence of the cycle skipping, amplitude and phase problems associated with the RFWI gradient. (a) RFWI gradient using full-offset range of data for both migration and inversion. (b) RFWI gradient using near-offset data for both migration and inversion. (c) RFWI gradient using the full-offset data for migration and near-offset data for inversion.

11 The (a) phase differences between (b) two shot gathers with trace mismatches. 
12 Comparison of reflection wavepaths between (a) conventional RFWI and (b) MRPI.

13 Comparison of the workflows between (a) conventional RFWI and (b) MRPI + MD method.

14 Comparison of the virtual source at the 1st iteration of (a) conventional FWI, (b) conventional RFWI and (c) MRPI + MD method.

15 The (a) true velocity model, (b) initial velocity model, (c) FWI gradient at the 1st iteration, (d) FWI tomogram, (e) RFWI gradient at the 1st iteration, (f) RFWI Tomogram, (g) MRPI gradient at the 1st iteration and (h) MRPI + MD tomogram.

16 The (a) true velocity model, (b) initial velocity model, (c) FWI gradient at the 1st iteration, (d) FWI tomogram, (e) RFWI gradient at the 1st iteration, (f) RFWI Tomogram, (g) MRPI gradient at the 1st iteration, and (h) MRPI + MD tomogram.

17 The comparisons of the velocity profiles in Figures 16(a), (b), (d), (f) and (h) at (a) $x=1 \mathrm{~km},(\mathrm{~b}) \mathrm{x}$ $=1.4 \mathrm{~km},(\mathrm{c}) \mathrm{x}=1.8 \mathrm{~km},(\mathrm{~d}) \mathrm{x}=2.2 \mathrm{~km},(\mathrm{e}) \mathrm{x}=2.6 \mathrm{~km}$ and (f) $\mathrm{x}=3.0 \mathrm{~km}$.

18 Comparisons of the migration images computed from (a) true velocity model, (b) initial velocity model, (c) FWI tomogram, (d) RFWI tomogram and (e) MRPI + MD tomogram.

19 The comparisons between the (a) raw shot gather, (b) processed shot gather, and (c) processed shot gather with three integrations. (d) The comparison of the normalized magnitude spectrum between (b) and (c).

20 Comparsions of the velocity models for the (a) initial model, (b) FWI tomogram, (c) RFWI tomogram and (d) MRPI + MD tomogram.

21 Comparsions of the migration images computed from the (a) initial model, (b) FWI tomogram, (c) RFWI tomogram and (d) MRPI + MD tomogram.

22 Comparsion of the sub-offset gathers computed from the (a) initial model and (b) MRPI + MD tomogram.

23 Comparsion of the angle gathers computed from the (a) initial model and (b) MRPI + MD tomo- 
gram.

24 Comparison between the observed data and Born data, where the rad and green wiggles represent the observed and Born data, respectively. 


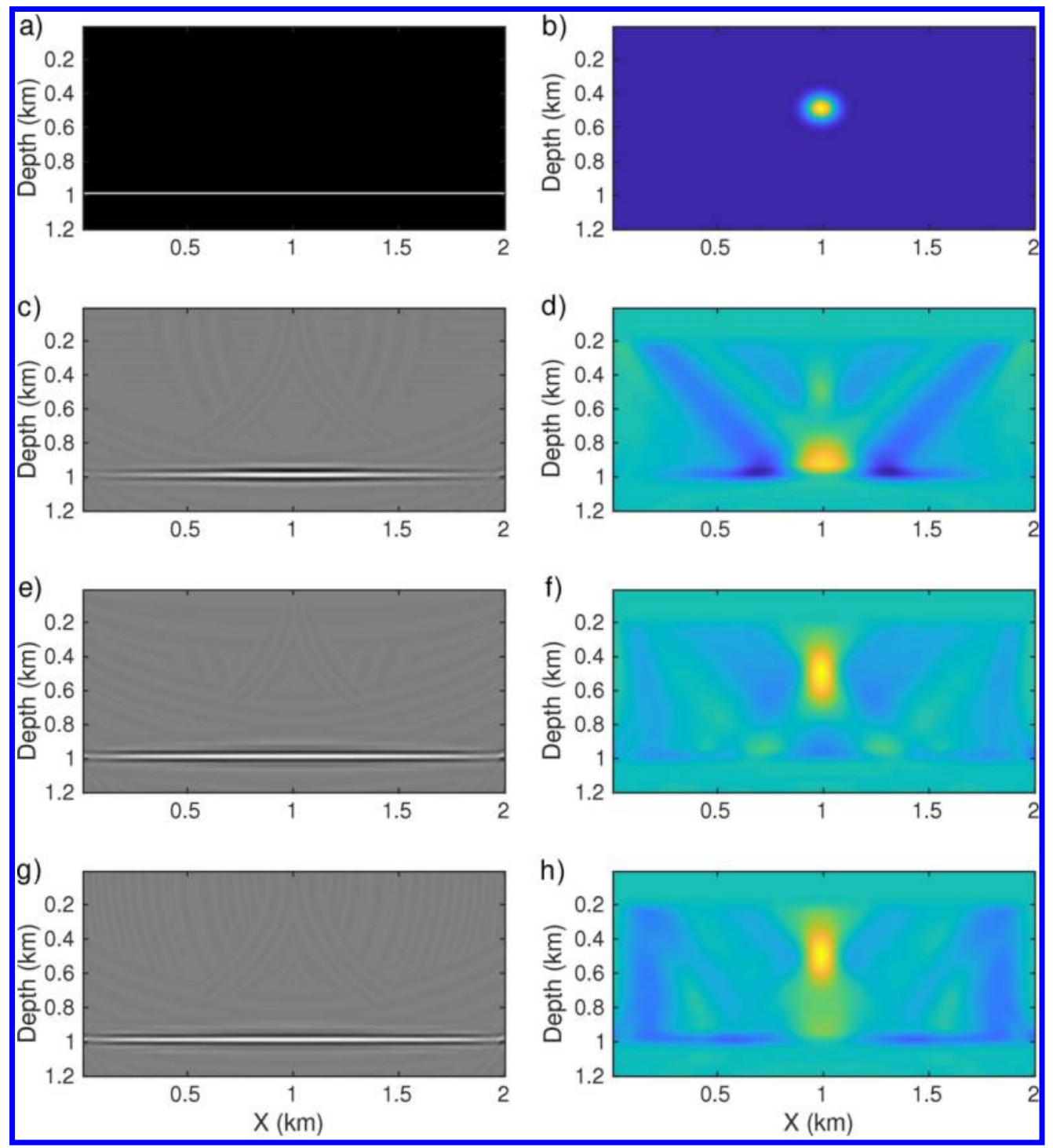

Figure 1 . The (a) true reflectivity model, (b) true velocity model, (c) reverse time migration (RTM) image, (e) least-squares reverse time migration (LSRTM) image and $(\mathrm{g})$ migration deconvolution (MD) image. The RFWI gradients based on the (d) RTM, (f) LSRTM, and (h) MD images.

$127 \times 140 \mathrm{~mm}(300 \times 300 \mathrm{DPI})$ 

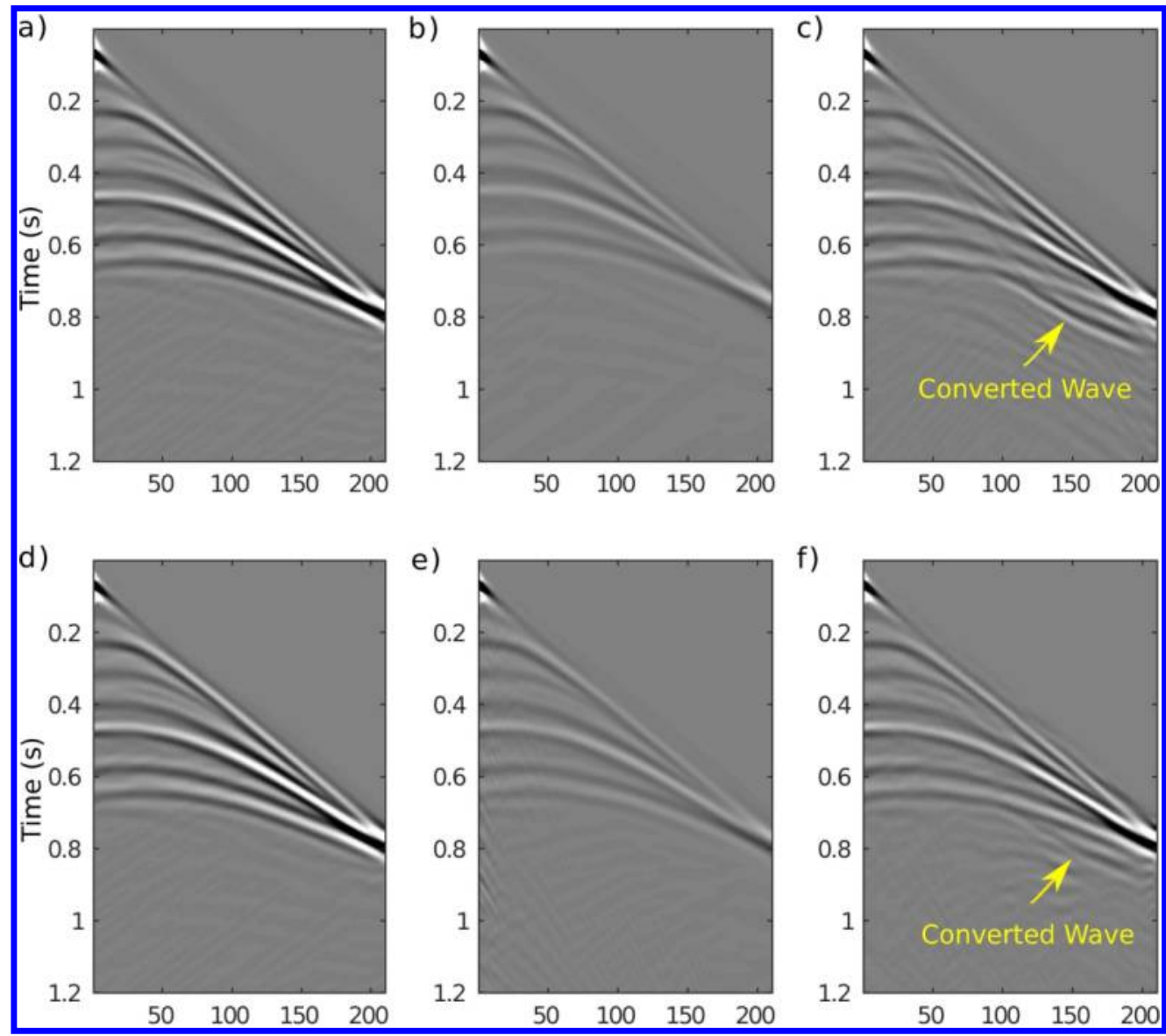

Figure2. The (a) acoustic, (b) viscoacoustic and (c) elastic data. The acoustic data replaced with (d) acoustic, (e) viscoacoustic and (f) elastic magnitude spectra, respectively.

$155 \times 137 \mathrm{~mm}(300 \times 300 \mathrm{DPI})$ 


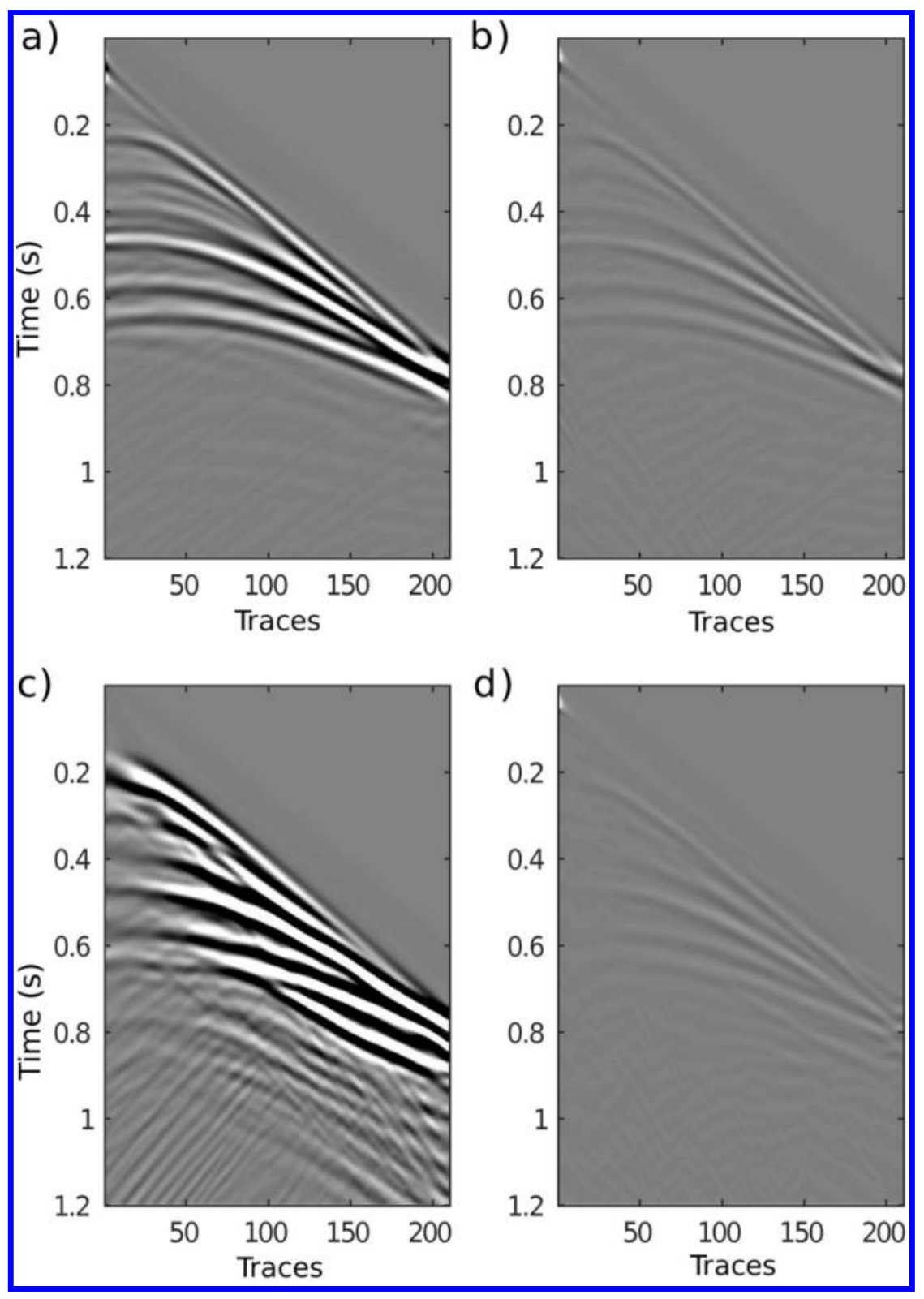

Figure3. The data residual between the acoustic data and visco data (a) before and (b) after magnitude spectra replacement. The data residual between the acoustic data and elastic data (c) before and (d) after magnitude spectra replacement.

$92 \times 130 \mathrm{~mm}(300 \times 300 \mathrm{DPI})$ 


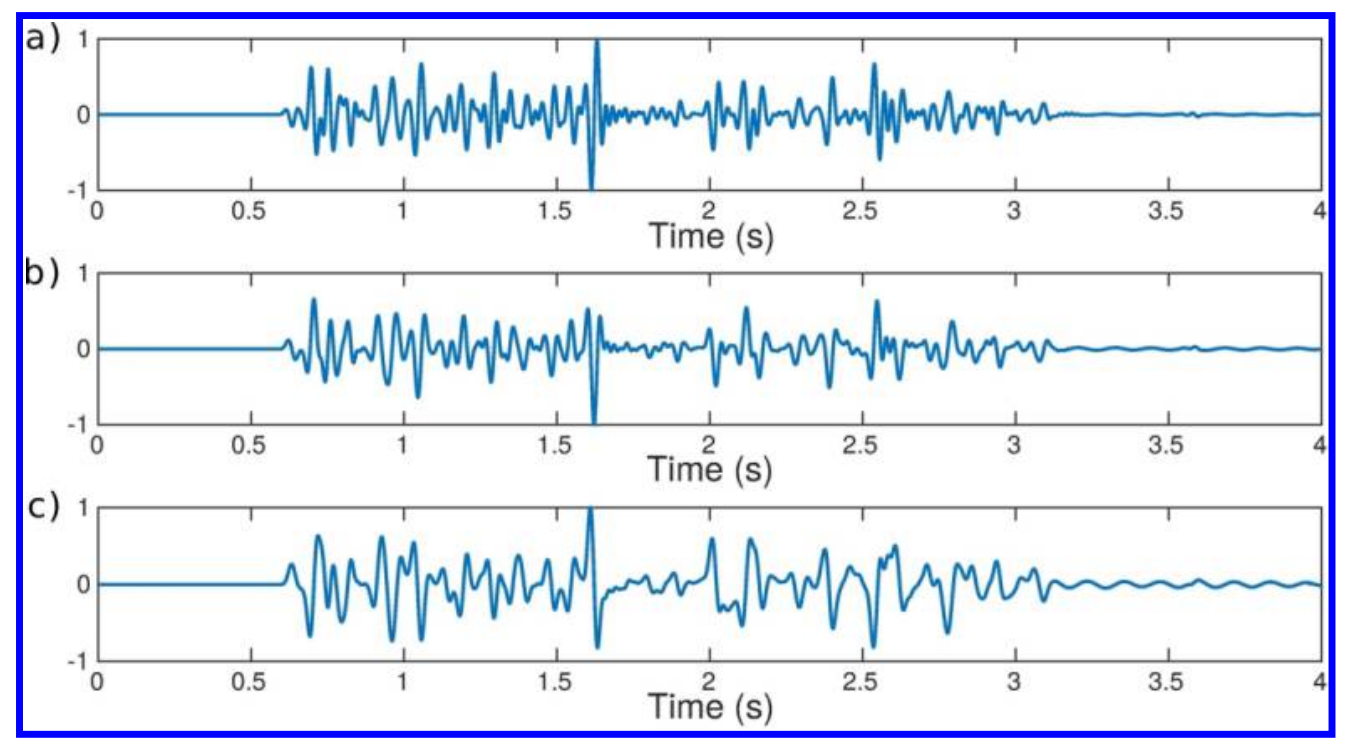

Figure4. The original trace (a) without integration, (b) with one and (c) two integration.

$145 \times 78 \mathrm{~mm}(300 \times 300 \mathrm{DPI})$

This paper presented here as accepted for publication in Geophysics prior to copyediting and composition. (c) 2020 Society of Exploration Geophysicists. 


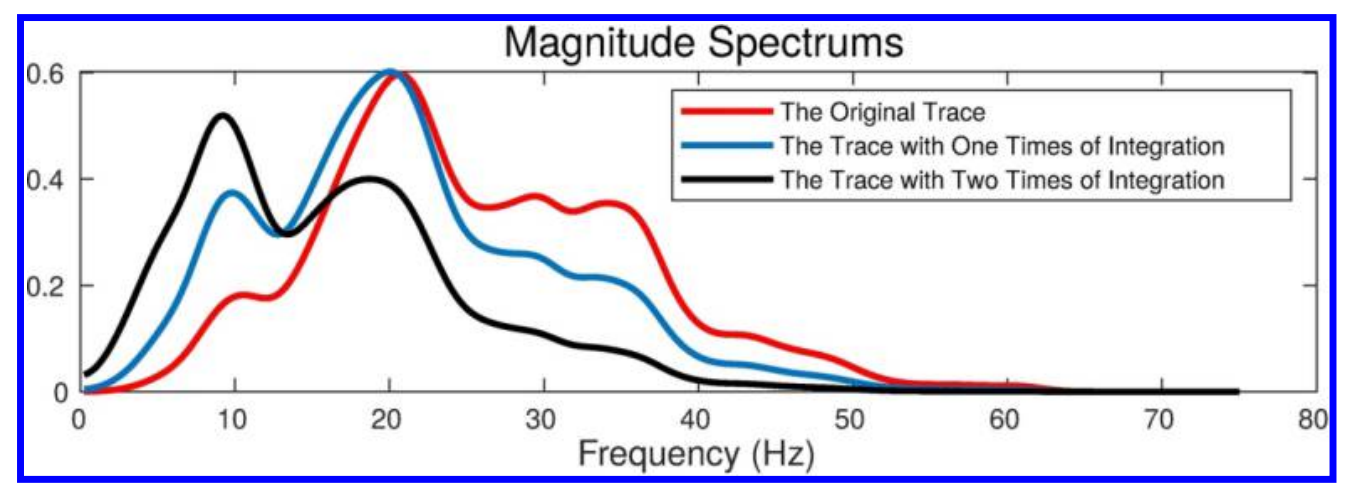

Figure5. The frequency spectrum of the original trace (a) without integration, with (b) one and (c) two integrations, which are represented by the red, blue and black curves, respectively.

$143 \times 50 \mathrm{~mm}(300 \times 300 \mathrm{DPI})$ 


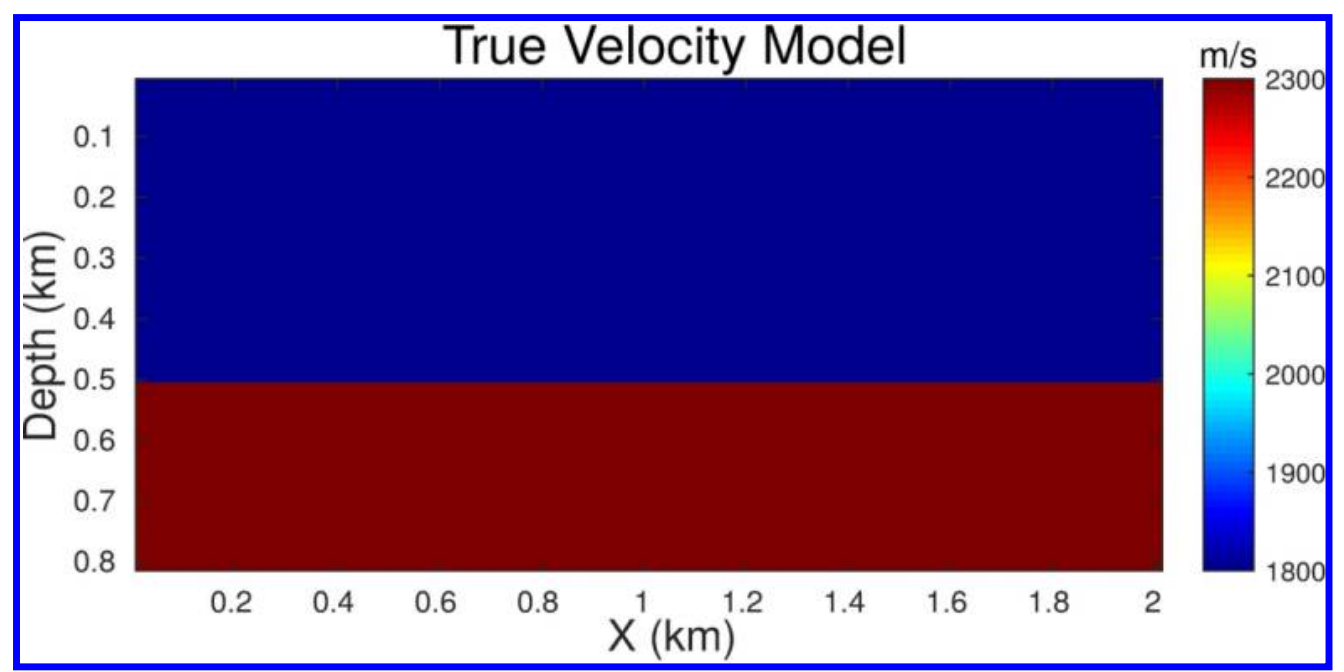

Figure6. Two-layer velocity model.

$147 \times 72 \mathrm{~mm}(300 \times 300 \mathrm{DPI})$ 

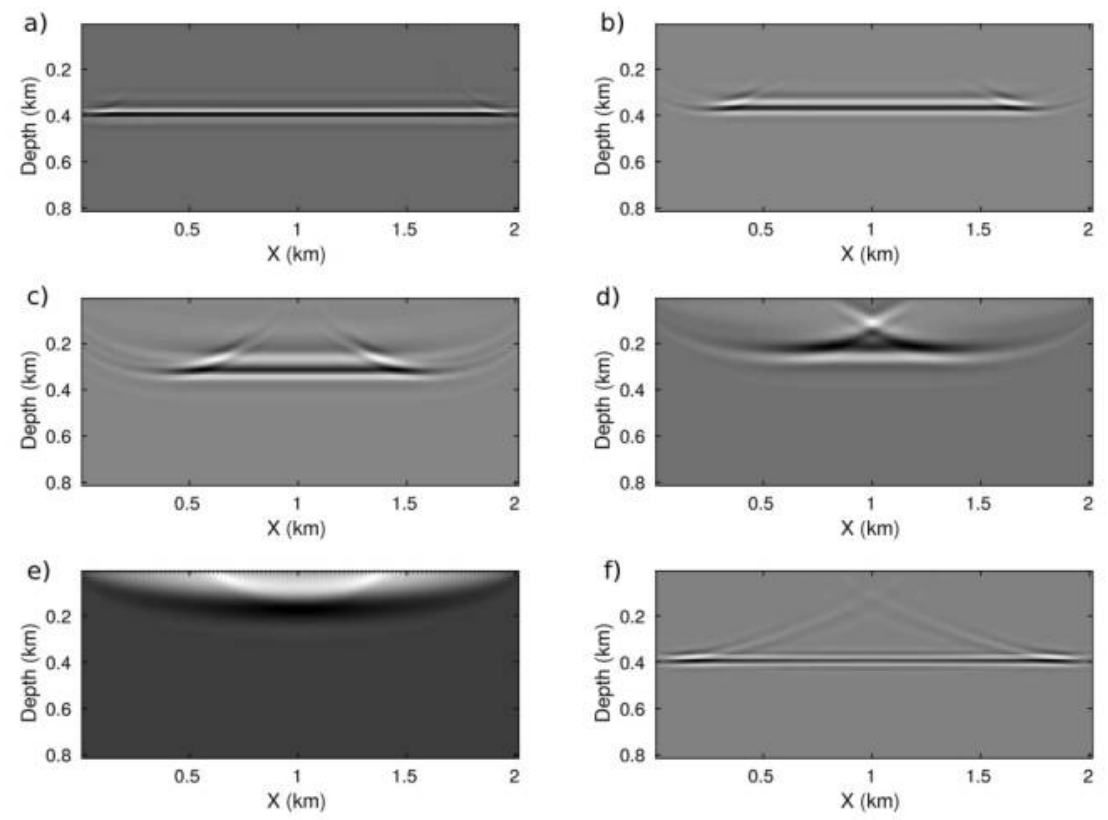

Figure7. Migration image with offset ranges of (a) less than $0.2 \mathrm{~km}$, (b) 0.3 to $0.5 \mathrm{~km}$, (c) 0.6 to $0.8 \mathrm{~km}$, (d) 0.9 to $1.1 \mathrm{~km},(\mathrm{e}) 1.2$ to $1.5 \mathrm{~km}$ and (f) the entire offset.

$226 \times 158 \mathrm{~mm}(300 \times 300 \mathrm{DPI})$ 


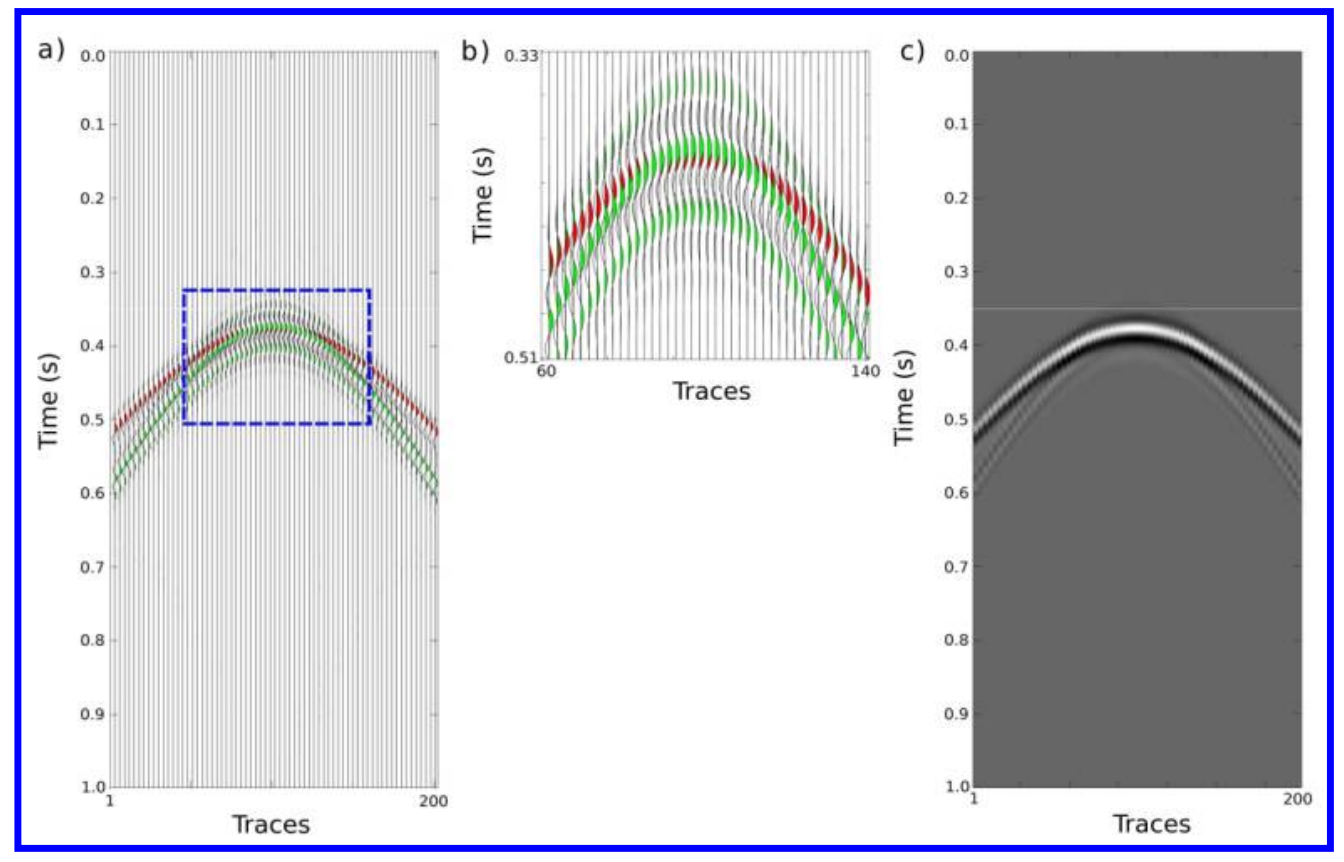

Figure8. The (a) trace comparisons between the observed data and Born data at $x=1.0 \mathrm{~km}$; (b) zoomed view of the blue box and (c) the data residuals.

$415 \times 263 \mathrm{~mm}(300 \times 300 \mathrm{DPI})$ 


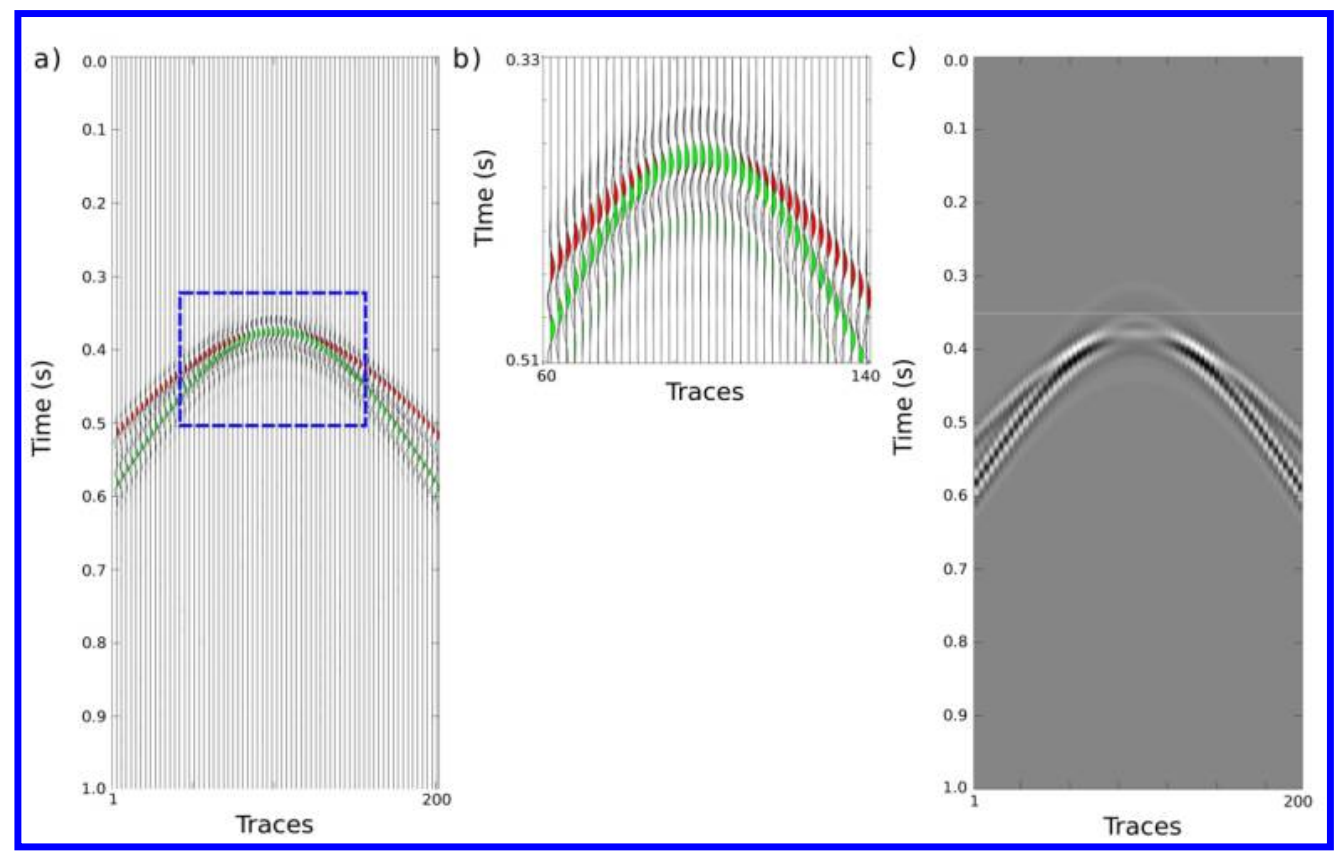

Figure9. The (a) trace comparison between the observed data and Born data at $x=1.0 \mathrm{~km}$; (b) zoomed view of the blue box and (c) the data residuals.

$415 \times 262 \mathrm{~mm}(300 \times 300 \mathrm{DPI})$ 


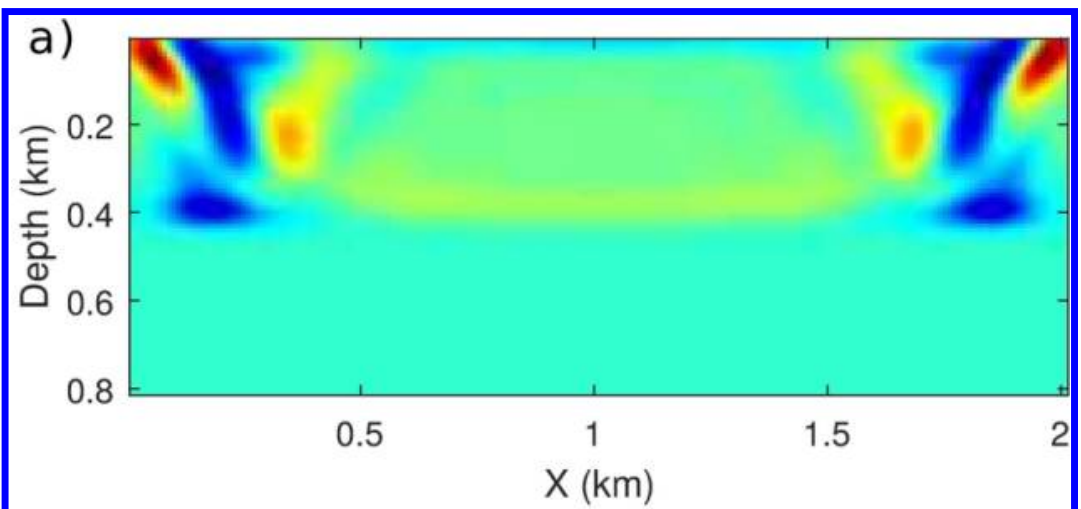

b)
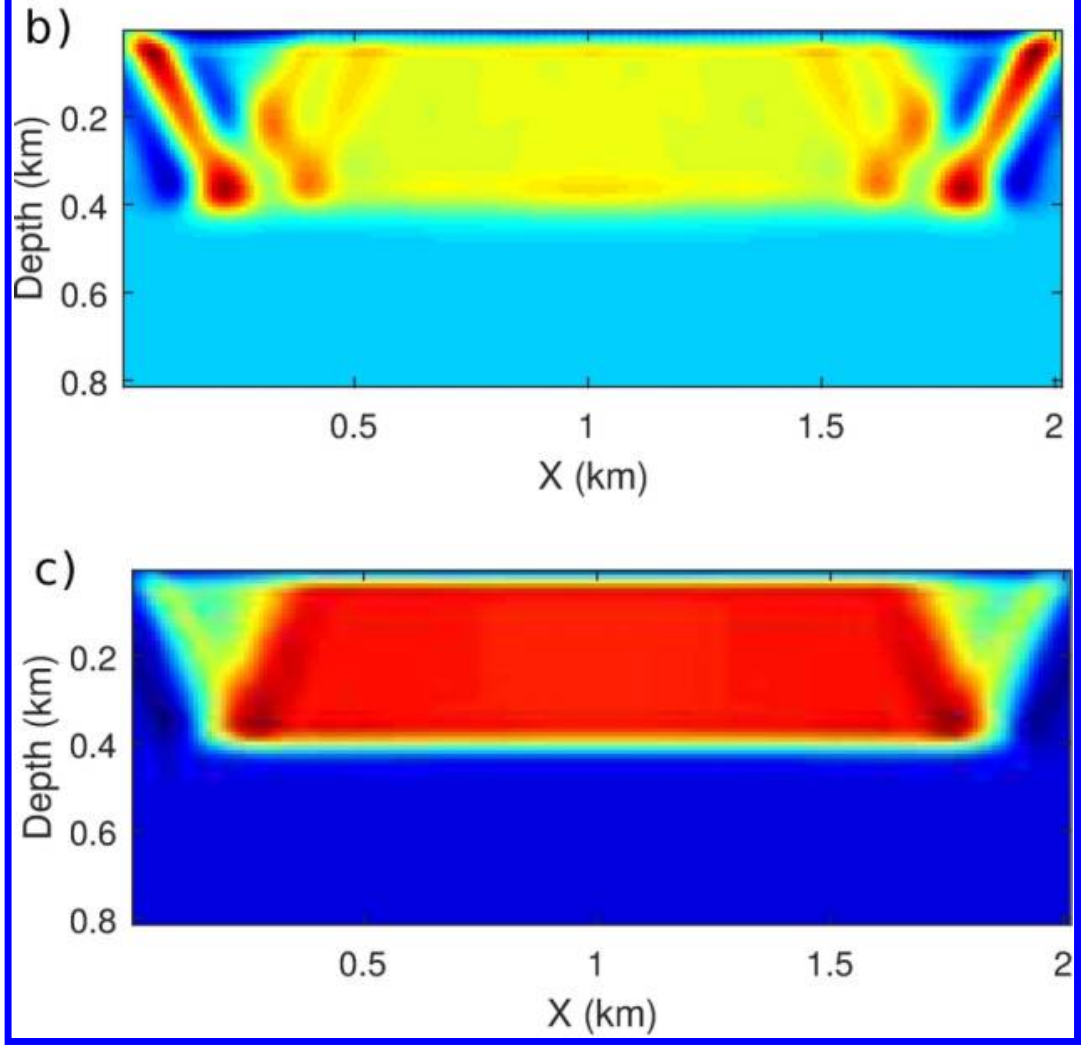

Figure10. Demonstration of the influence of the cycle skipping, amplitude and phase problems associated with the RFWI gradient. (a) RFWI gradient using a full-offset range of data for both migration and inversion. (b) RFWI gradient using near-offset data for both migration and inversion. (c) RFWI gradient using the fulloffset data for migration and near-offset data for inversion.

$$
108 \times 160 \mathrm{~mm}(300 \times 300 \mathrm{DPI})
$$




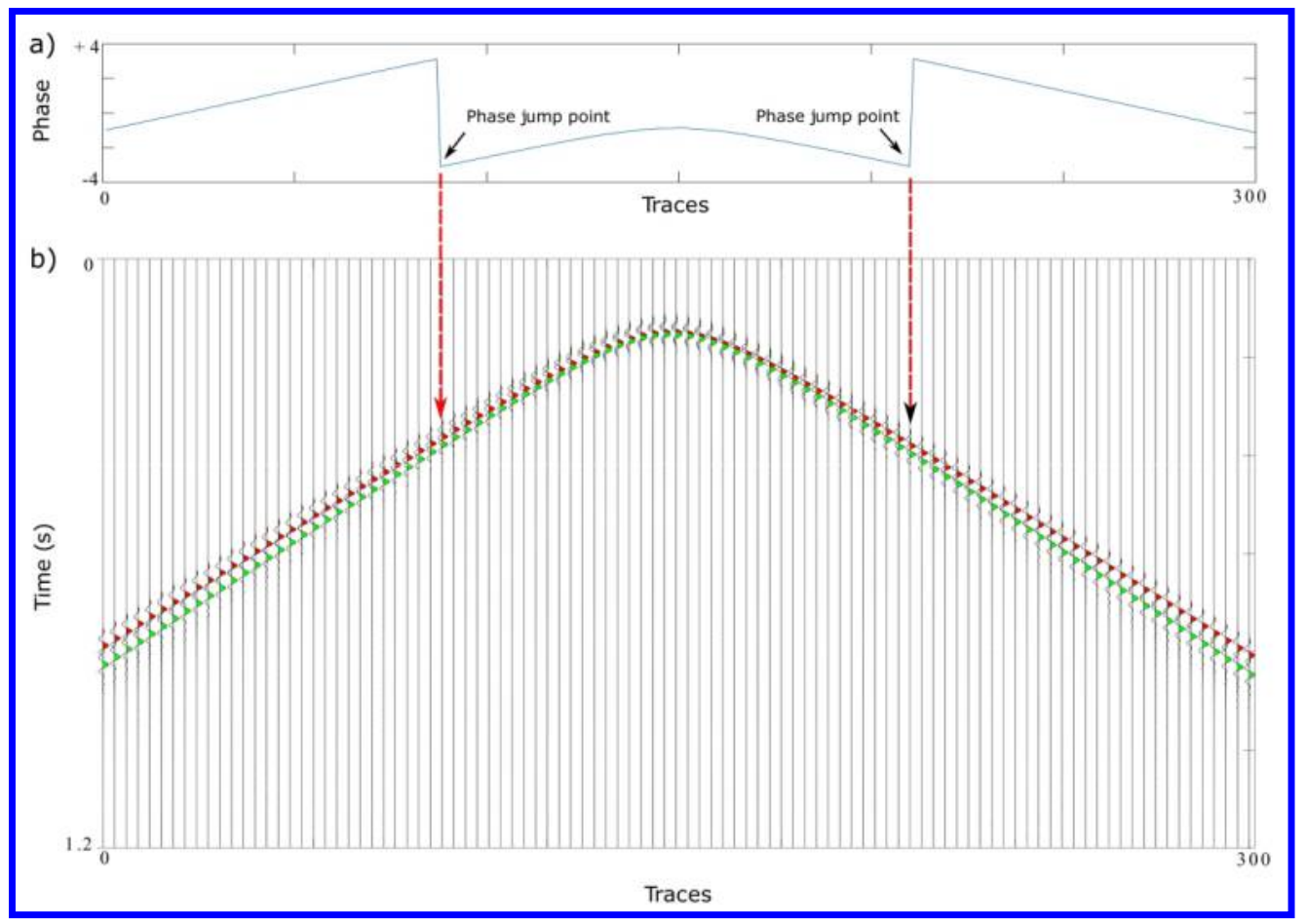

Figure11. The (a) phase differences between (b) two shot gather with trace mismatches.

$379 \times 266 \mathrm{~mm}(300 \times 300 \mathrm{DPI})$

This paper presented here as accepted for publication in Geophysics prior to copyediting and composition.

(C) 2020 Society of Exploration Geophysicists. 


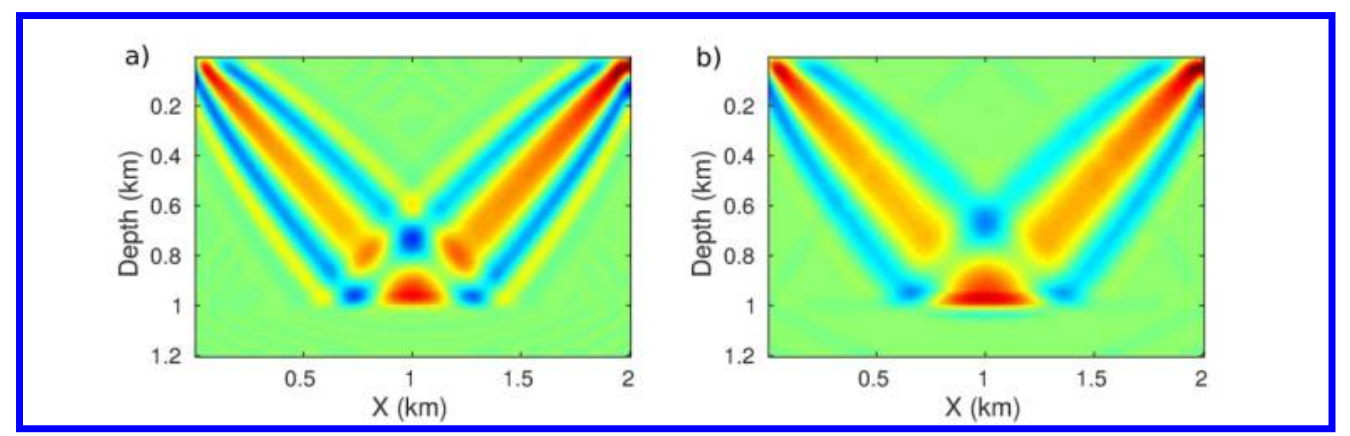

Figure12. Comparison of reflection wavepaths between (a) conventional RFWI and (b) MRPI. $195 \times 60 \mathrm{~mm}(300 \times 300 \mathrm{DPI})$ 


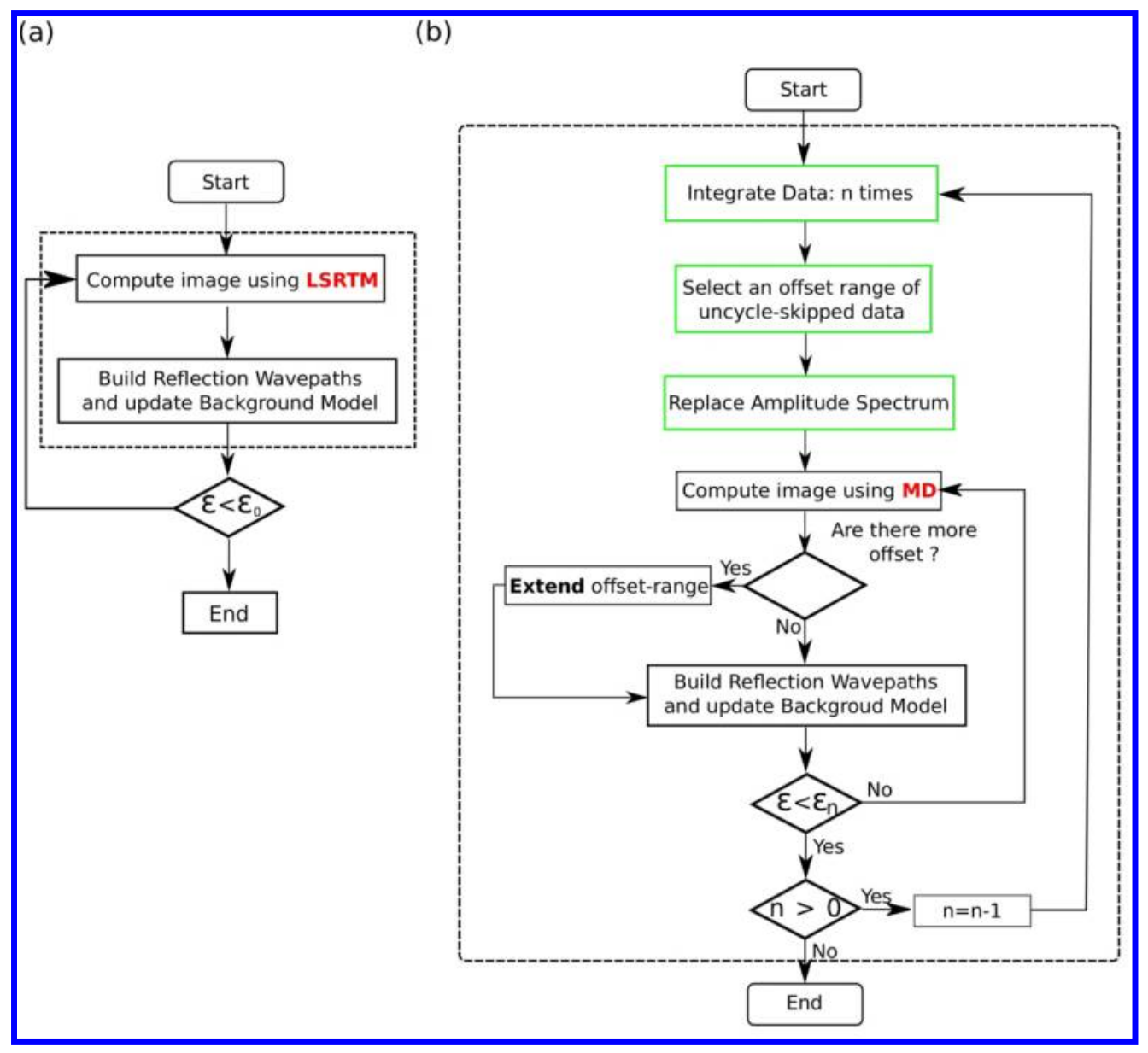

Figure13. Comparison of the workflows between (a) conventional RFWI and (b) MRPI + MD method. $202 \times 185 \mathrm{~mm}(300 \times 300$ DPI $)$ 


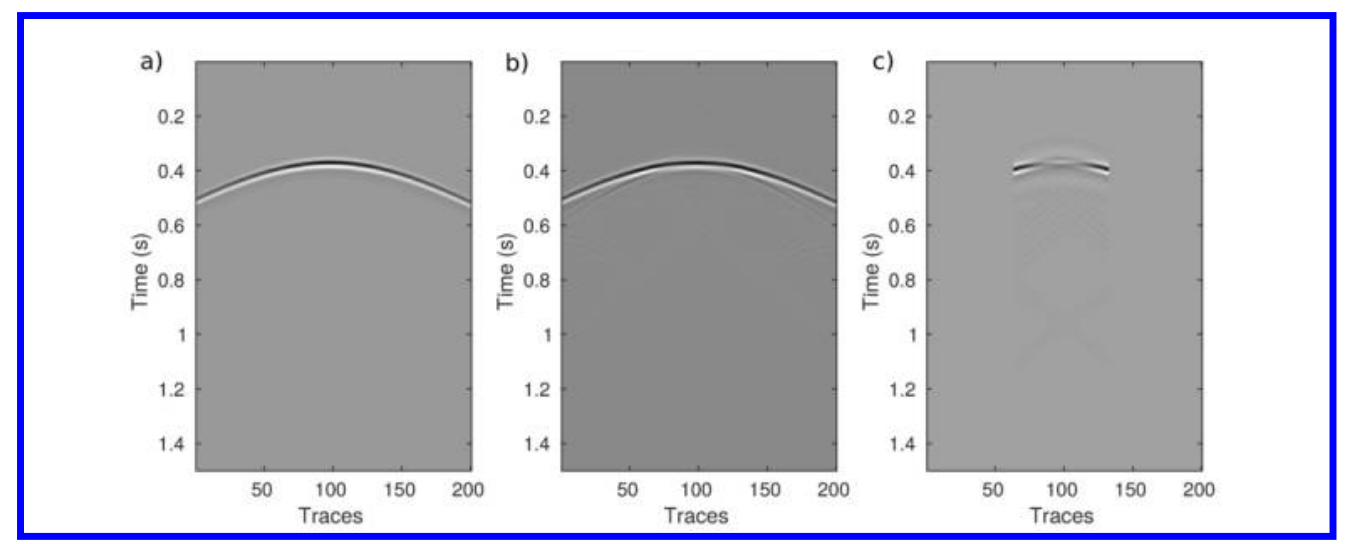

Figure14. Comparison of the virtual source at the 1st iteration of (a) conventional FWI, (b) conventional RFWI and (c) MRPI + MD method.

\section{$214 \times 83 \mathrm{~mm}(300 \times 300 \mathrm{DPI})$}




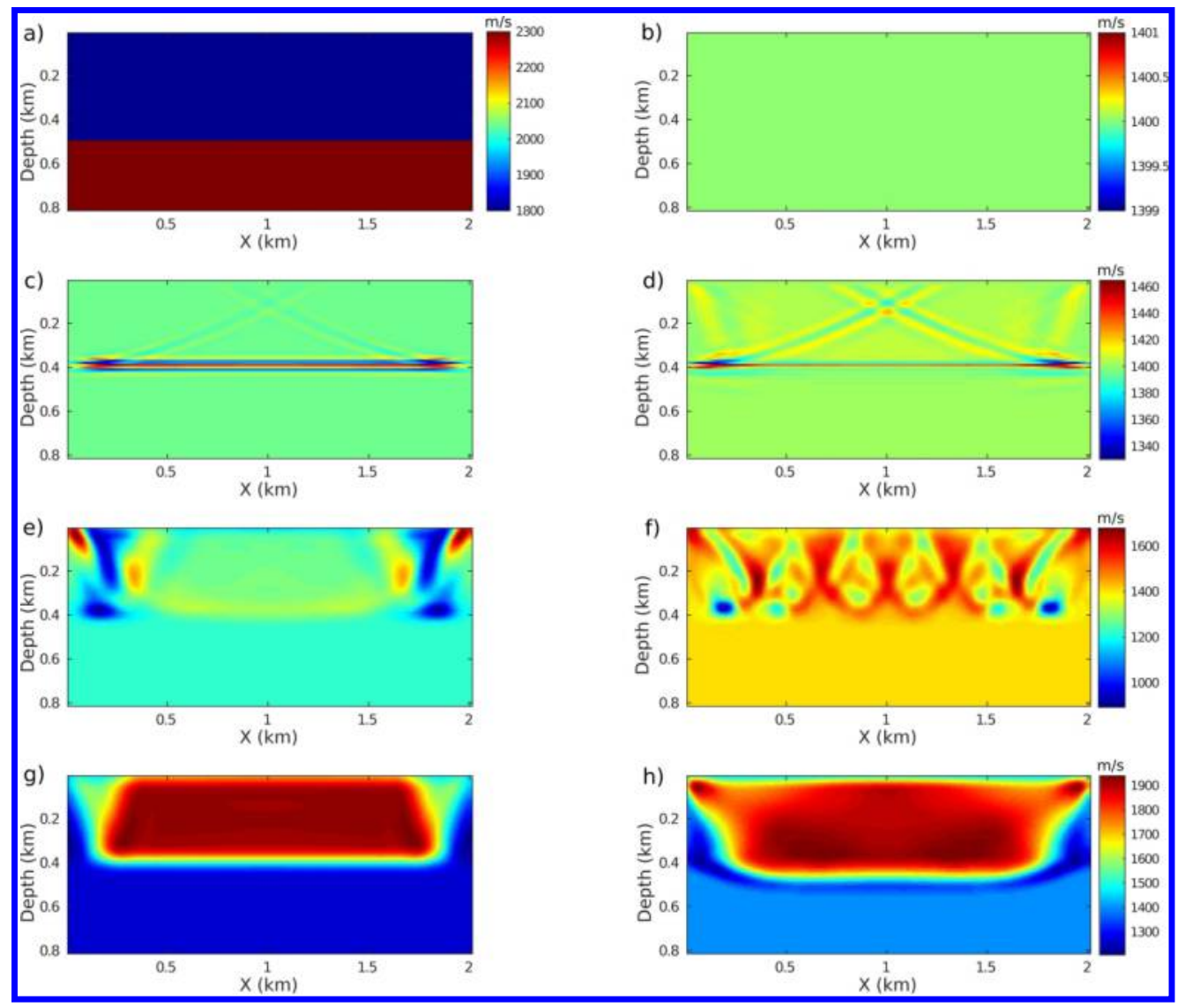

Figure15. The (a) true velocity model, (b) initial velocity model, (c) FWI gradient at the 1st iteration, (d) FWI tomogram, (e) RFWI gradient at the 1st iteration, (f) RFWI Tomogram, (g) MRPI gradient at the 1st iteration and (h) MRPI + MD tomogram.

$265 \times 226 \mathrm{~mm}(300 \times 300 \mathrm{DPI})$ 


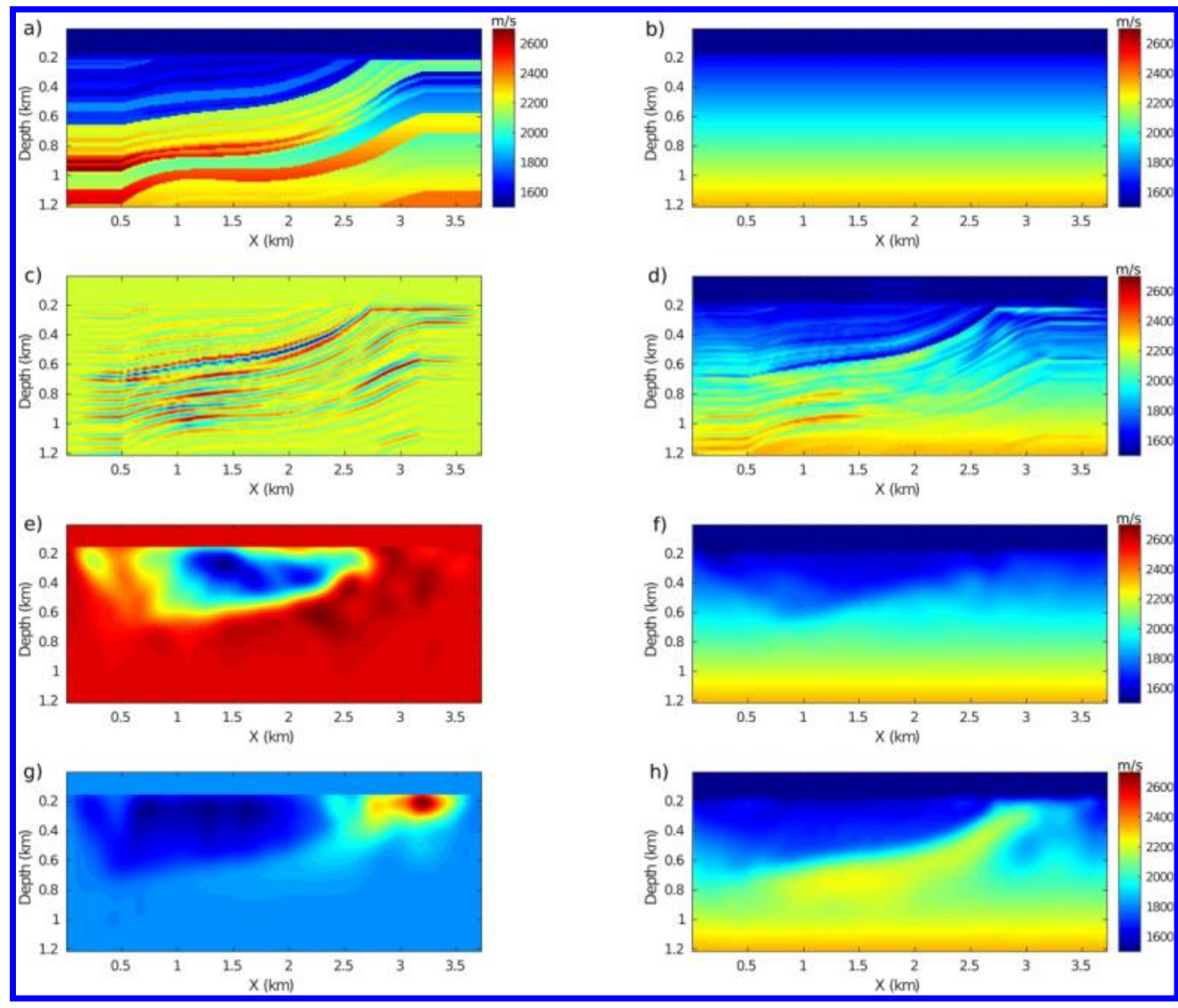

Figure16. The (a) true velocity model, (b) initial velocity model, (c) FWI gradient at the 1st iteration, (d) FWI tomogram, (e) RFWI gradient at the 1st iteration, (f) RFWI Tomogram, (g) MRPI gradient at the 1st iteration, and (h) MRPI + MD tomogram.

\section{$295 \times 250 \mathrm{~mm}(300 \times 300 \mathrm{DPI})$}



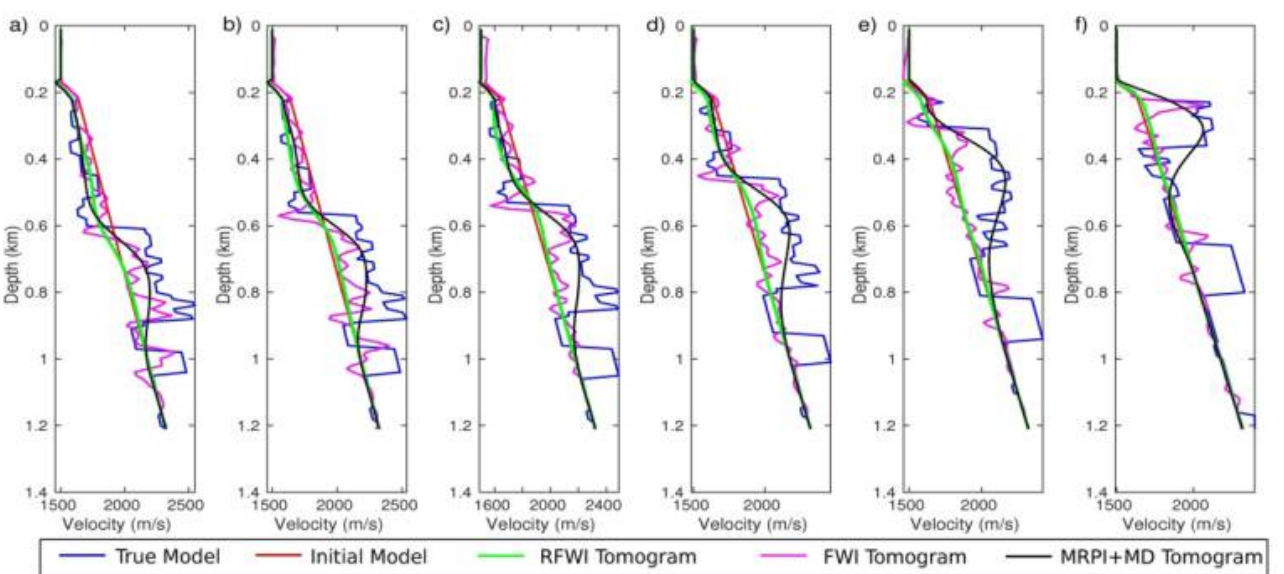

Figure17. The comparisons of the velocity profiles in Figures ef $\{$ fig:fig8\}(a), (b), (d), (f) and (h) at (a) $x=$ $1 \mathrm{~km},(\mathrm{~b}) \mathrm{x}=1.4 \mathrm{~km},(\mathrm{c}) \mathrm{x}=1.8 \mathrm{~km}$, (d) $x=2.2 \mathrm{~km},(\mathrm{e}) \mathrm{x}=2.6 \mathrm{~km}$ and (f) $x=3.0 \mathrm{~km}$.

$$
237 \times 110 \mathrm{~mm}(300 \times 300 \mathrm{DPI})
$$

This paper presented here as accepted for publication in Geophysics prior to copyediting and composition. (C) 2020 Society of Exploration Geophysicists. 


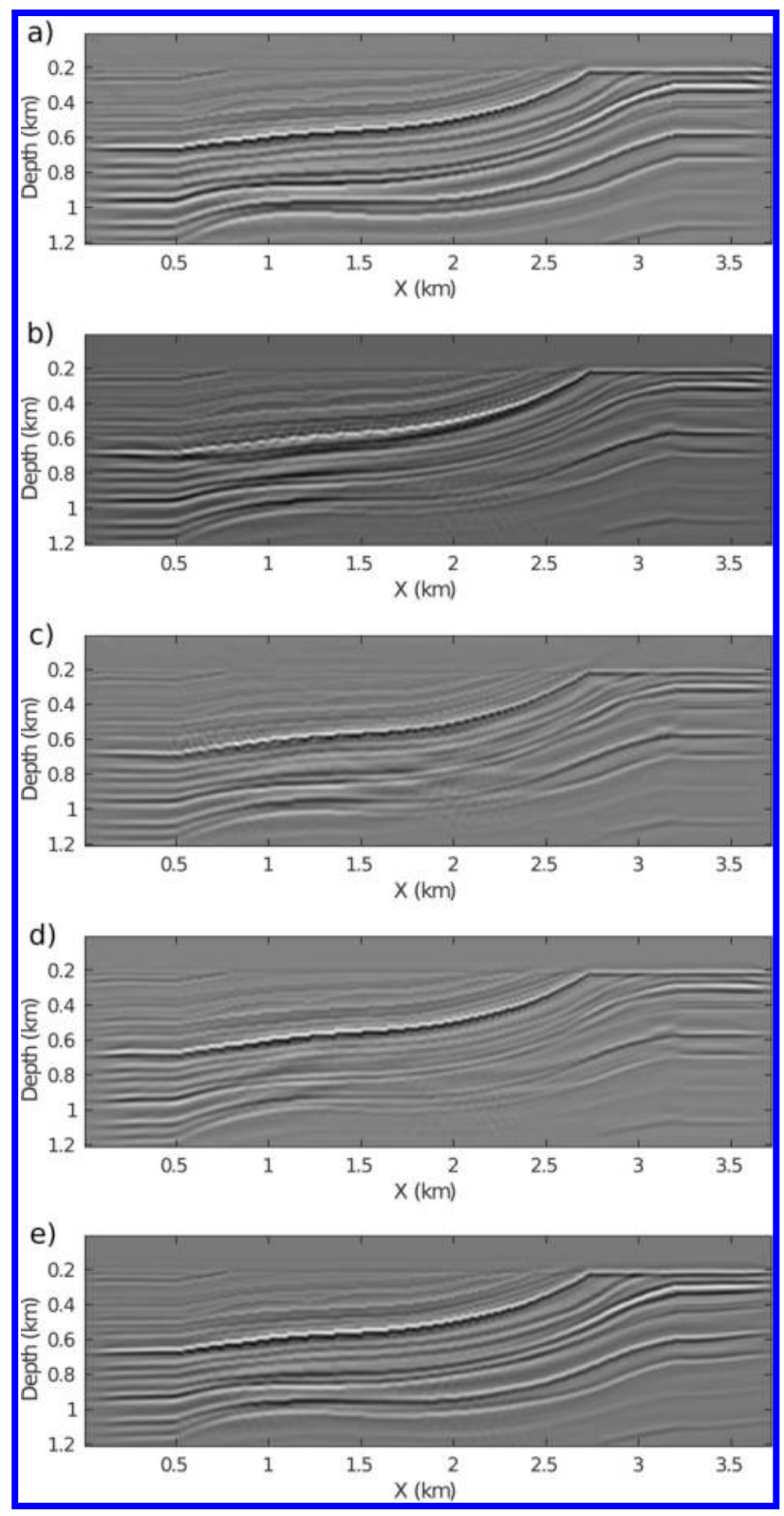

Figure18. Comparisons of the migration images computed from (a) true velocity model, (b) initial velocity model, (c) FWI tomogram, (d) RFWI tomogram and (e) MRPI + MD tomogram.

$$
125 \times 246 \mathrm{~mm}(300 \times 300 \mathrm{DPI})
$$




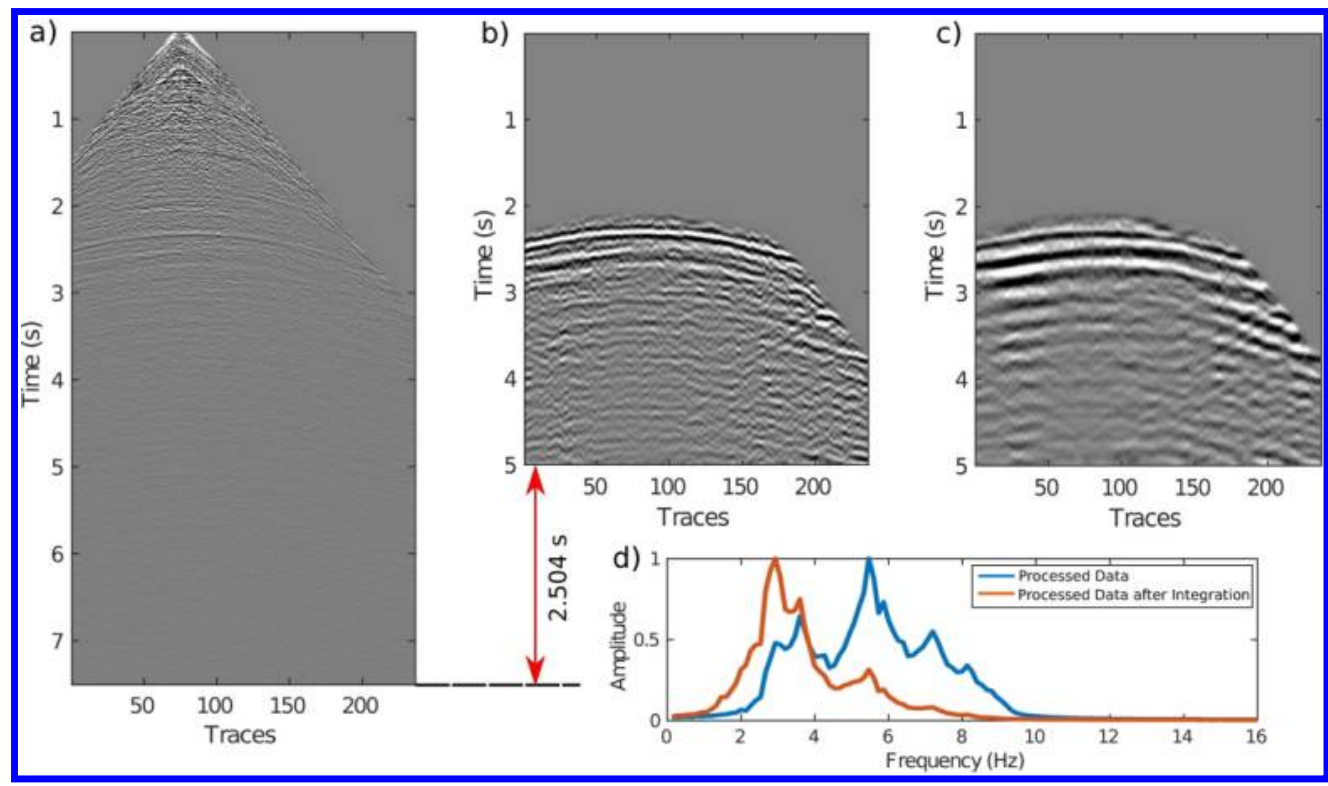

Figure19. The comparisons between the (a) raw shot gather, (b) processed shot gather, and (c) processed shot gather with three integrations. (d) The comparison of the normalized magnitude spectrum between (b) and (c).

$192 \times 111 \mathrm{~mm}(300 \times 300 \mathrm{DPI})$ 

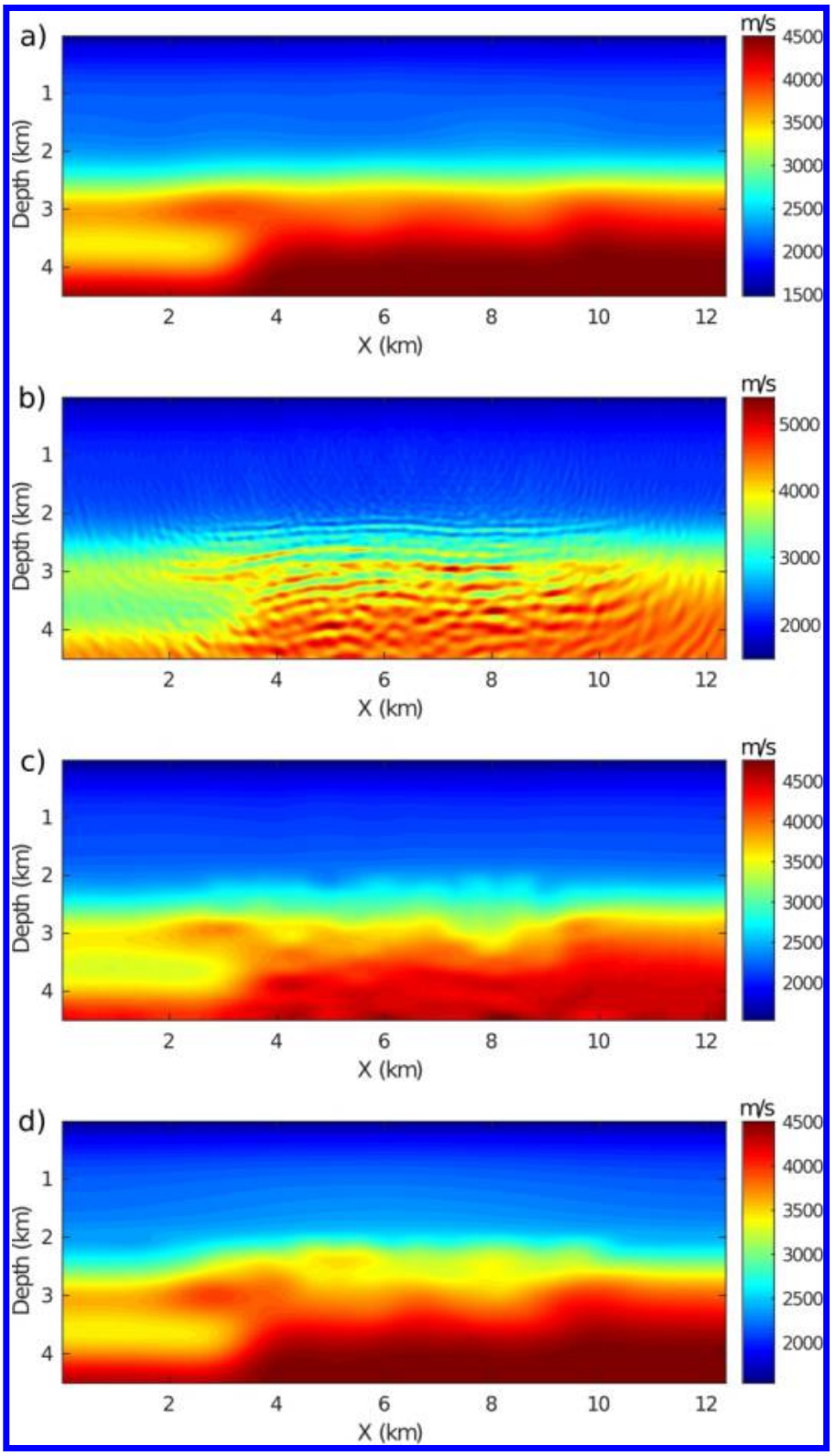

Figure20. Comparisons of the velocity models for the (a) initial model, (b) FWI tomogram, (c) RFWI tomogram and (d) MRPI + MD tomogram.

$142 \times 250 \mathrm{~mm}(300 \times 300 \mathrm{DPI})$ 


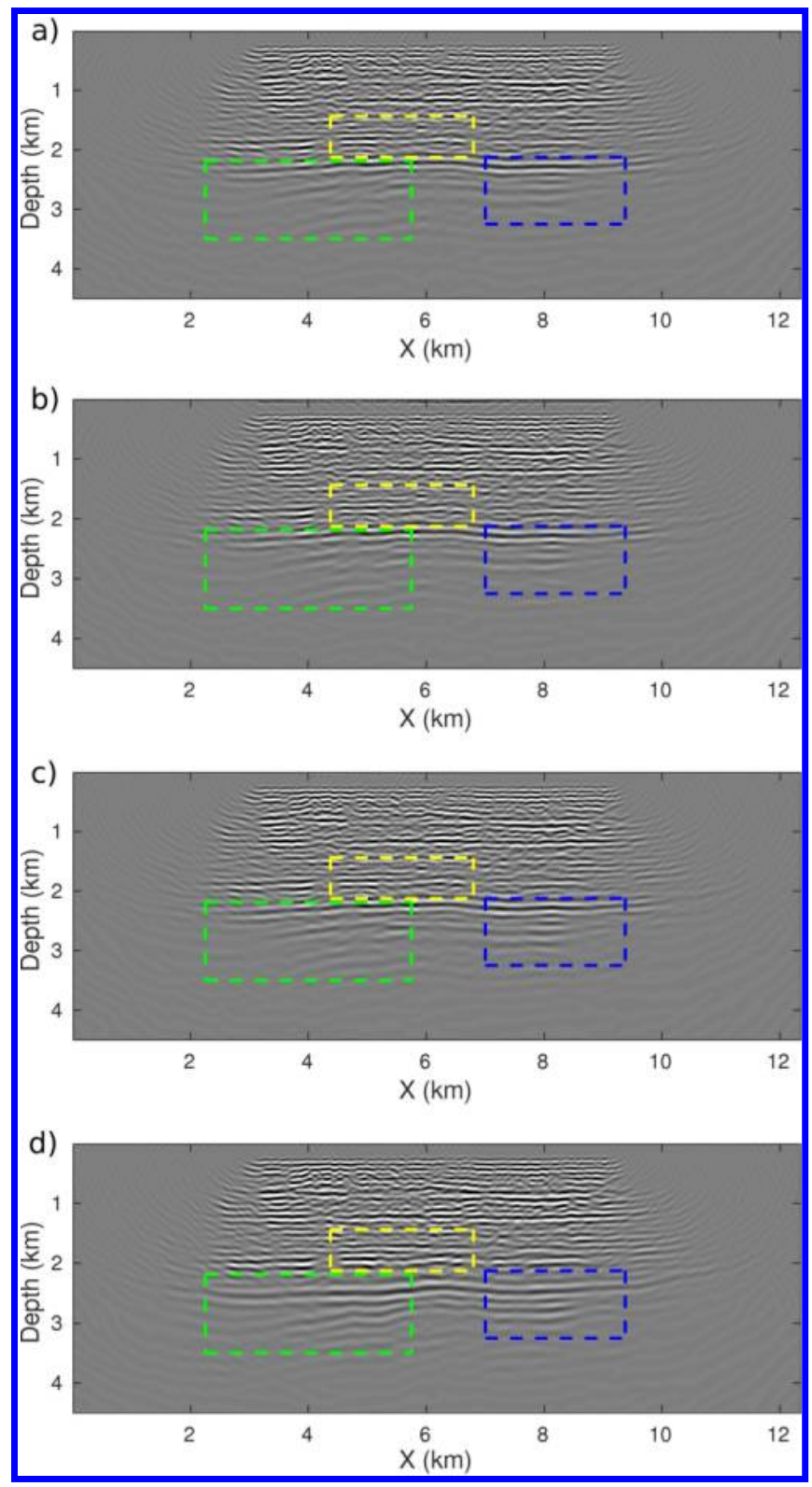

Figure21. Comparisons of the migration images computed from the (a) initial model, (b) FWI tomogram, (c) RFWI tomogram and (d) MRPI + MD tomogram.

$133 \times 248 \mathrm{~mm}(300 \times 300 \mathrm{DPI})$ 


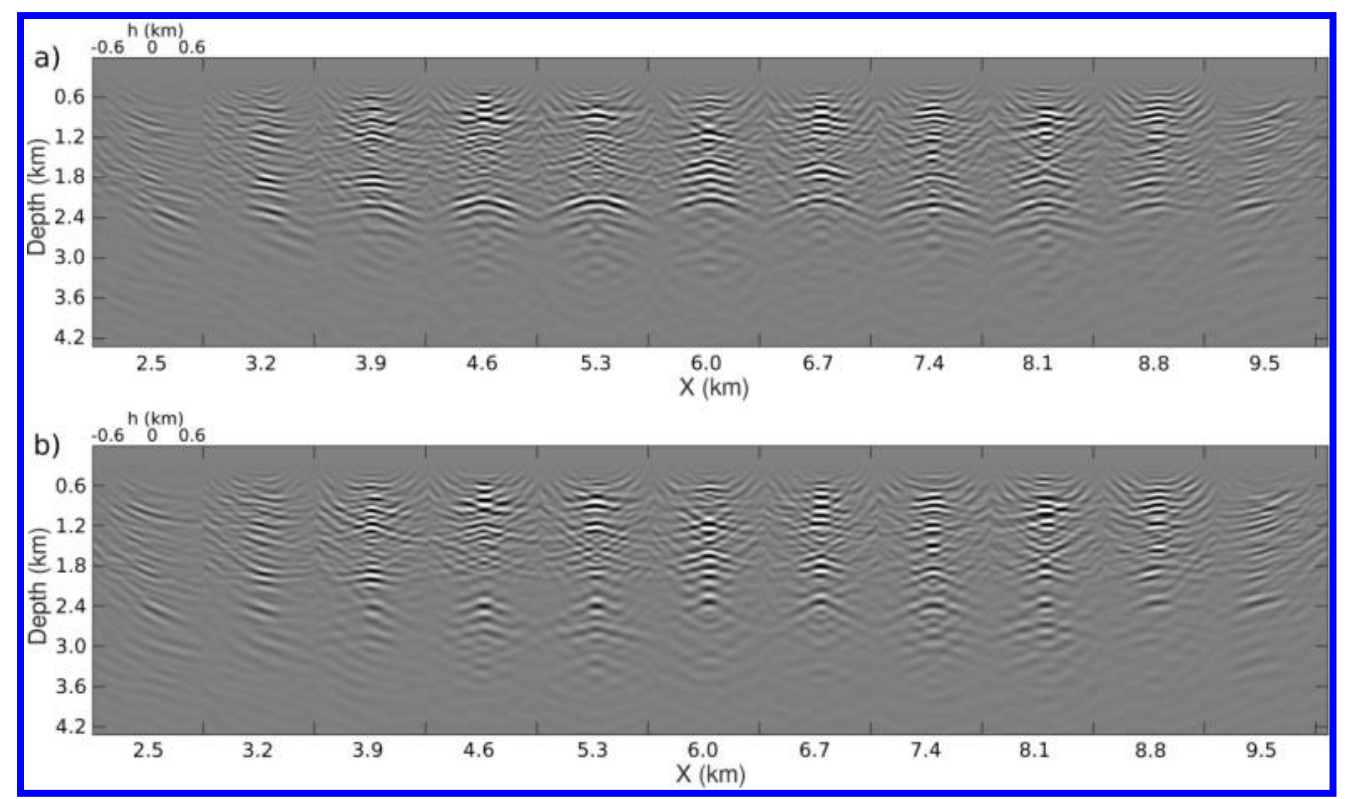

Figure22. Comparison of the sub-offset gathers computed from the (a) initial model and (b) MRPI + MD tomogram.

$266 \times 157 \mathrm{~mm}(300 \times 300 \mathrm{DPI})$

This paper presented here as accepted for publication in Geophysics prior to copyediting and composition. (C) 2020 Society of Exploration Geophysicists. 


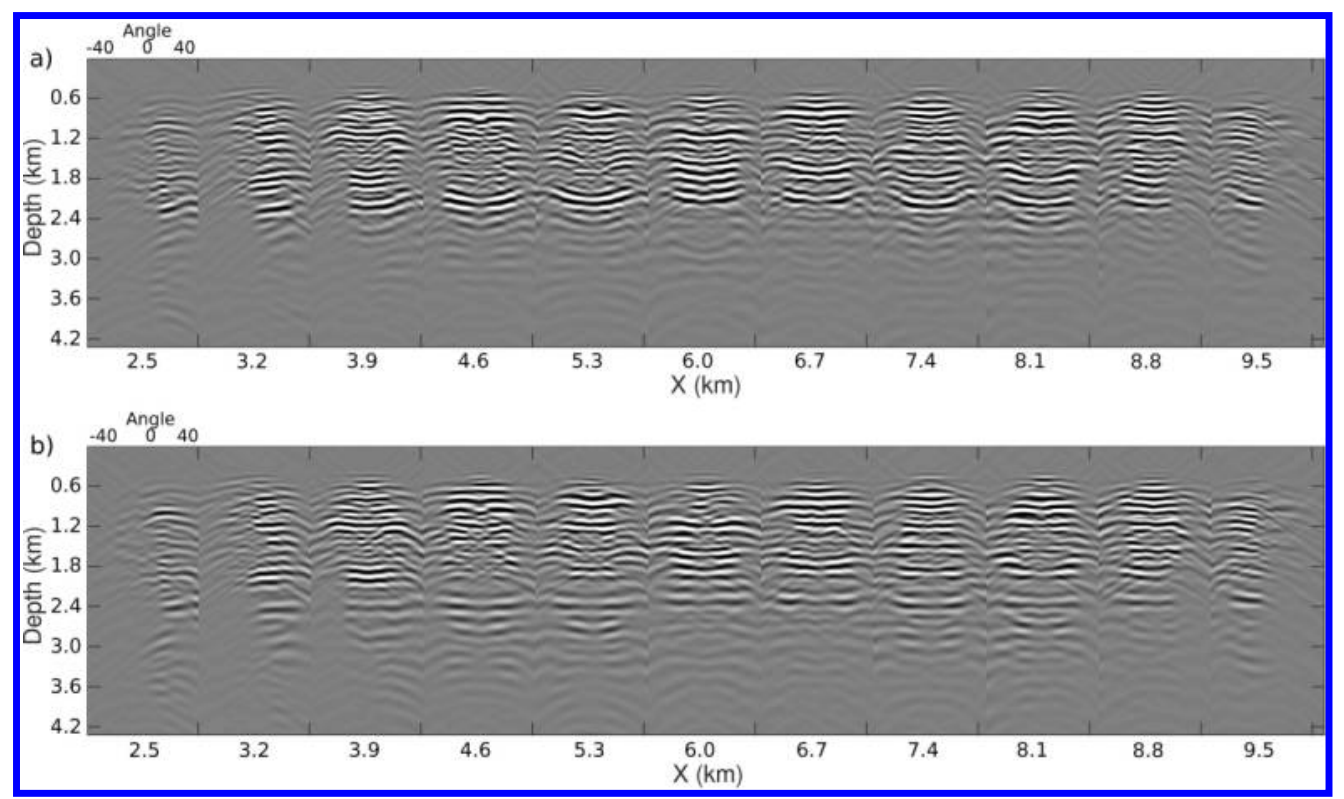

Figure23. Comparison of the angle gathers computed from the (a) initial model and (b) MRPI + MD tomogram.

$266 \times 156 \mathrm{~mm}(300 \times 300$ DPI)

This paper presented here as accepted for publication in Geophysics prior to copyediting and composition.

(C) 2020 Society of Exploration Geophysicists. 


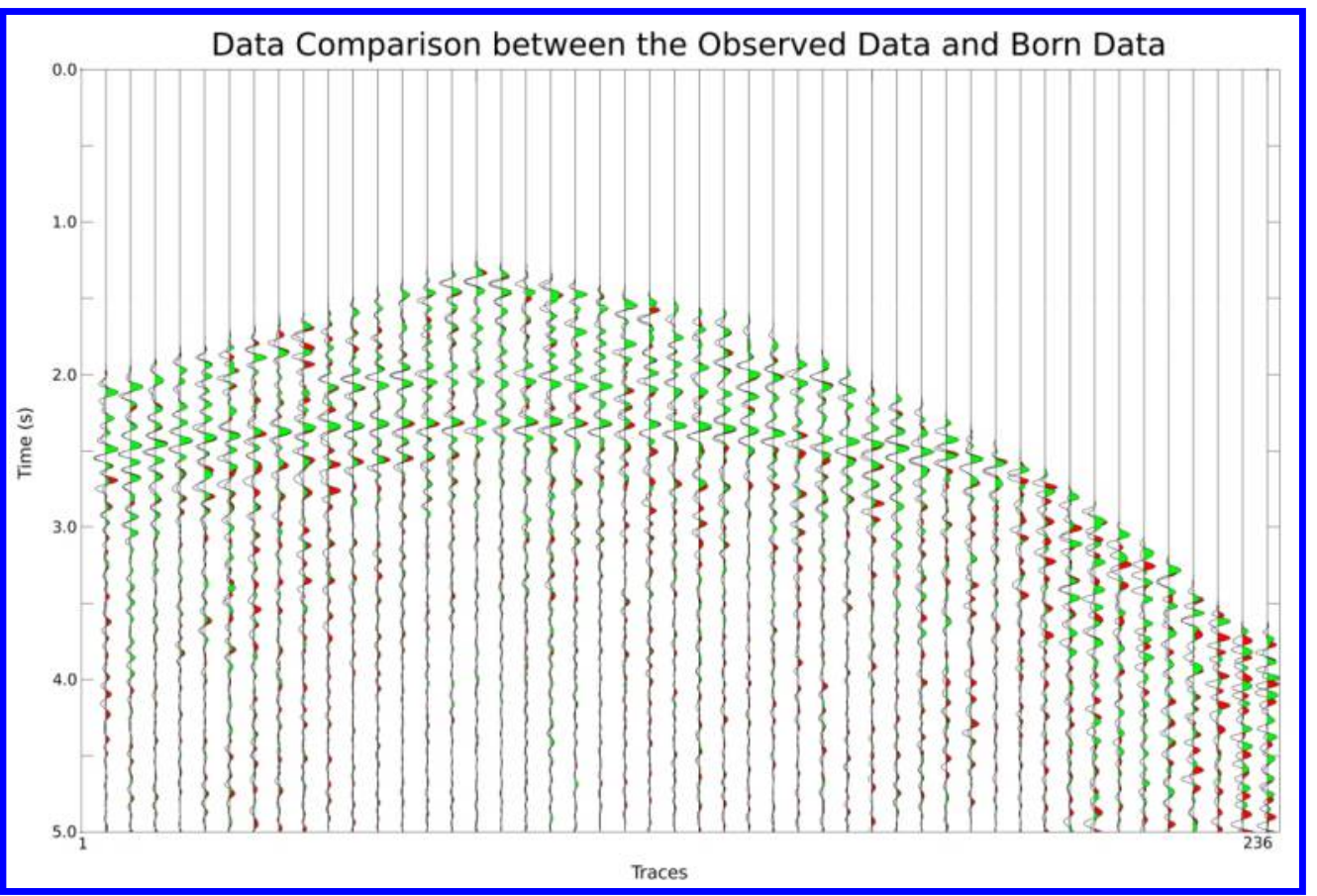

Figure24. Comparison between the observed data and Born data, where the rad and green wiggles represent the observed and Born data, respectively.

$408 \times 275 \mathrm{~mm}(300 \times 300 \mathrm{DPI})$

This paper presented here as accepted for publication in Geophysics prior to copyediting and composition. (C) 2020 Society of Exploration Geophysicists. 
DATA AND MATERIALS AVAILABILITY

Data associated with this research are confidential and cannot be released. 
This article has been cited by:

1. Lei Fu, Zongcai Feng, Gerard T. Schuster. 2019. Multiscale phase inversion for 3D ocean-bottom cable data. Geophysical Prospecting 202. . [Crossref] 